Accepted for publication in ApJ, May 152007

\title{
Physical and Spectral Characteristics of the T8 and Later-Type Dwarfs
}

\author{
S. K. Leggett \\ Gemini Observatory, 670 N. A'ohoku Place Hilo HI 96720 \\ M. S. Marley \\ MS 245-3, NASA Ames Research Center, Moffett Field, CA 94035 \\ R. Freedman \\ SETI, 515 N. Whisman Road, Mountain View, CA 94043 \\ D. Saumon \\ Los Alamos National Laboratory, MS F663, Los Alamos, NM 87545 \\ dsaumon@lanl.gov \\ Michael C. Liu \\ Alfred P. Sloan Research Fellow, Institute for Astronomy, University of Hawaii, 2680 \\ Woodlawn Drive, Honolulu HI 96822 \\ T. R. Geballe \\ Gemini Observatory, 670 N. A'ohoku Place, Hilo HI 96720 \\ D. A. Golimowski \\ Department of Physics 83 Astronomy, Johns Hopkins University, 3400 North Charles \\ Street, Baltimore, MD 21218 \\ and \\ D. C. Stephens \\ BYU Department of Physics \&3 Astronomy, N-145 ESC, Provo, UT 84602
}

\section{ABSTRACT}


We use newly observed and published near-infrared spectra, together with synthetic spectra obtained from model atmospheres, to derive physical properties of three of the latest-type $\mathrm{T}$ dwarfs. $\mathrm{A}$ new $\mathrm{R} \approx 1700$ spectrum of the T7.5 dwarf HD 3651B, together with existing data, allows a detailed comparison to the wellstudied and very similar dwarf, Gl 570D. We find that HD 3651B has both higher gravity and metallicity than Gl 570D, with best-fit atmospheric parameters of $T_{\text {eff }}=820-830 \mathrm{~K}, \log g=5.4-5.5,[\mathrm{~m} / \mathrm{H}]=+0.2$ and $K_{z z}=10^{4} \mathrm{~cm}^{2} \mathrm{~s}^{-1}$. Its age is $8-12$ Gyr and its implied mass is $60-70 \mathrm{M}_{\text {Jupiter }}$. We perform a similar analyis of the T8 and T7.5 dwarfs 2MASS J09393548-2448279 and 2MASS J111451332618235 using published data, comparing them to the well-studied T8, 2MASS J04151954-0935066. We find that these two dwarfs have effectively the same $T_{\text {eff }}$ as the reference dwarf, and similar or slightly higher gravities, but lower metallicities. The derived parameters are $T_{\text {eff }}=725-775 \mathrm{~K}$ and $[\mathrm{m} / \mathrm{H}]=-0.3$; $\log g=5.3-5.45$ for 2MASS J09393548-2448279 and $\log g=5.0-5.3$ for 2MASS J11145133-261823. The age and mass are 10 Gyr and $60 \mathrm{M}_{\mathrm{Jupiter}}$ for 2MASS J09393548-2448279, and 5 Gyr and $40 \mathrm{M}_{\text {Jupiter }}$ for 2MASS J11145133-261823.

A serious limitation to such analyses is the incompleteness of the line lists for transitions of $\mathrm{CH}_{4}$ and $\mathrm{NH}_{3}$ at $\lambda \leq 1.7 \mu \mathrm{m}$, which are also needed for synthesizing the spectrum of the later, cooler, Y type. Spectra of Saturn and Jupiter, and of laboratory $\mathrm{CH}_{4}$ and $\mathrm{NH}_{3}$ gas, suggest that $\mathrm{NH}_{3}$ features in the $Y$ - and $J$ bands may be useful as indicators of the next spectral type, and not features in the $H$ - and $K$-bands as previously thought. However, until cooler objects are found, or the line lists improve, large uncertainties remain, as the abundance of $\mathrm{NH}_{3}$ is likely to be significantly below the chemical equilibrium value. Moreover inclusion of laboratory $\mathrm{NH}_{3}$ opacities in our models predicts band shapes that are discrepant with existing data. It is possible that the $\mathrm{T}$ spectral class will have to be extended to temperatures around $400 \mathrm{~K}$, when water clouds condense in the atmosphere and dramatically change the spectral energy distribution of the brown dwarf.

Subject headings: infrared: stars - stars: low-mass, brown dwarfs - stars: individual (2MASS J04151954-0935066, 2MASS J09393548-2448279, 2MASS J111451332618235, HD 3651B, Gl 570D) 


\section{Introduction}

The first of the T- or methane-type ultracool brown dwarfs, Gl 229B, was discovered as a companion to an M dwarf by Nakajima et al. in 1995. It took four more years for other examples to be found, and these were revealed primarily as a result of far-red and near-infrared large area sky surveys: the Sloan Digital Sky Survey (SDSS; York et al. 2000) and the Two Micron All Sky Survey (2MASS; Beichman et al. 1998, Skrutskie et al. 2006). There are now over 100 spectroscopically confirmed and published T dwarfs (see the online compendium at DwarfArchives.org). A near-infrared classification scheme for $\mathrm{T}$ dwarfs, using $\mathrm{H}_{2} \mathrm{O}$ and $\mathrm{CH}_{4}$ absorption bands, has been defined by the 2MASS and SDSS groups (Burgasser et al. 2006a). Gl 229B is now classified as a peculiar T7 dwarf, and the other known $\mathrm{T}$ dwarfs range from $\mathrm{T} 0$ to $\mathrm{T} 8$.

In this paper we study three of the six known dwarfs later than $\mathrm{T} 7$ published as of February 2007. A summary of the astrometric properties and previously derived physical parameters of all six is given in Table 1. The uncertainty in spectral type classification is typically 0.5 of a subclass; hence by including T7.5 dwarfs this sample is representative of a "T8" sample. New and fainter very-late T dwarfs are now being discovered in the UKIRT Infrared Deep Sky Survey (UKIDSS; Hewett et al. 2006, Lawrence et al. 2007, Warren et al. 2007a). Lodieu et al. (2007) present eight new T dwarfs, of which the latest is classified as a T7.5. Warren et al. (2007b) present the latest-type T dwarf to date, a T8.5 dwarf. These two dwarfs are not discussed further, and the reader is directed to the discovery papers.

Three of the six dwarfs listed in Table 1, 2MASS J04151954-0935066 (T8, Burgasser et

al. 2002, hereafter referred to as 2MASS0415-09), 2MASS J12171110-0311131 (T7.5, Burgasser et al. 1999, hereafter 2MASS1217-03) and Gl 570D (T7.5, Burgasser et al. 2000), have been recently examined in detail by Saumon et al. (2006, 2007) using synthetic spectra calculated from model atmospheres, and ground- and space-based observational data covering the wavelength range 0.9-15 $\mu \mathrm{m}$. Saumon et al. (2007) show that 2MASS121703 is more metal-rich and hotter than the other two $\mathrm{T}$ dwarfs, with effective temperature $T_{\text {eff }} \approx 900 \mathrm{~K}$. Gl $570 \mathrm{D}$ has an effective temperature of $T_{\text {eff }} \approx 800 \mathrm{~K}$ and 2 MASS0415-09 has a lower $T_{\text {eff }}$ of around $750 \mathrm{~K}$. In this paper we use Gl 570D and 2MASS0415-09 as reference objects for the other three dwarfs listed in Table 1, 2MASS J09393548-2448279 and 2MASS J11145133-2618235 (Tinney et al. 2005, hereafter 2MASS0939-24 and 2MASS1114-26), and HD 3651B (Murgauer et al. 2006, Luhman et al. 2007).

Burgasser, Burrows \& Kirkpatrick (2006b, hereafter BBK) present a method for determining the physical parameters of the coldest $\mathrm{T}$ dwarfs using low-resolution near-infrared spectra. BBK use an index measuring the strength of the $\mathrm{H}_{2} \mathrm{O}$ feature at $1.15 \mu \mathrm{m}, \mathrm{H}_{2} \mathrm{O}-J$, as a measure of $T_{\text {eff }}$, while the ratio of the $2.1 \mu \mathrm{m}$ flux to the $1.6 \mu \mathrm{m}$ flux, $K / H$, is used to 
measure gravity. They use modelled values of these ratios, calibrated by the $T_{\text {eff }}$ and gravity of the well-studied dwarf Gl 570D, to determine $T_{\text {eff }}$ and gravity for a sample of T6 to T8 dwarfs. The values for the dwarfs in our sample are given in Table 1. BBK suggest that 2MASS0939-24 and 2MASS1114-26 are the coolest dwarfs known, with $T_{\text {eff }}$ s cooler than that of 2MASS0415-09 by $\gtrsim 50 \mathrm{~K}$. However, the gravity index of BBK is also sensitive to metallicity, as shown for example by Liebert \& Burgasser (2007) and Liu, Leggett \& Chiu (2007, hereafter LLC). This could affect the conclusions drawn by BBK; thus here we re-examine the properties of these two very-late $\mathrm{T}$ dwarfs.

We also examine in detail the atmospheric properties of HD 3651B. Luhman et al. (2007), Burgasser (2007) and LLC use near-infrared spectra and (for Luhman et al.) IRAC 3-8 $\mu \mathrm{m}$ photometry, to determine the physical parameters of this dwarf. The values of LLC and BBK are given in Table 1 (the LLC values agree well with those of Luhman et al.). This dwarf is of particular interest, as its metallicity and age can be constrained from its primary star, which is only possible for one other of the very late T dwarfs, Gl 570D. HD 3651B is also of interest as it is a wide companion to a star which hosts a planet (Fischer et al. 2003).

Finally, as several groups continue to search for dwarfs later and cooler than these (e.g. UKIDSS), we also explore what such objects may look like, using the observed spectra of the solar system gas giant planets Jupiter and Saturn, and laboratory spectra of $\mathrm{CH}_{4}$ and $\mathrm{NH}_{3}$ gas.

\section{Known Properties of the Sample}

\subsection{The K V + T7.5 Systems: Gl 570 and HD 3651}

Figure 1 shows previously published low-resolution near-infrared spectra for the T7.5 dwarfs Gl 570D and HD 3651B. It can be seen that the spectra are quite similar, with HD 3651B being slightly brighter in the $1.6 \mu \mathrm{m} H$-band and more significantly brighter in the $2.1 \mu \mathrm{m} K$-band.

The Gl 570 system consists of a $\mathrm{K}$ dwarf, two early-M dwarfs, and the $\mathrm{T}$ dwarf. The last was discovered in the 2MASS database by Burgasser et al. (2000) as a very widely separated 258".3 (1525 AU projected separation) companion to the system. HD 3651B is also a companion to a $\mathrm{K}$ dwarf, at a distance of 42.9 (476 AU projected separation), and the primary is known to be an exoplanet host. HD 3651B was discovered in near- and midinfrared imaging searches for companions to exoplanet stars by Mugrauer et al. (2006) and Luhman et al. (2007). The metallicities of both primaries are known: Gl 570A has $[\mathrm{m} / \mathrm{H}]=$ +0.00 to +0.07 (Feltzing \& Gustafsson 1998, Santos et al. 2005), and HD 3651A has $[\mathrm{m} / \mathrm{H}]=$ 
+0.09 to +0.16 (Gray et al. 2003, Santos et al. 2004, Valenti \& Fischer 2005).

Evolutionary models, constrained by the observed luminosity and further refined by comparison to synthetic spectra, show that the age of Gl 570D is $3-5 \mathrm{Gyr}$, with $T_{\text {eff }}=$ $800-820 \mathrm{~K}, \log g=5.09-5.23$ and mass $38-47 \mathrm{M}_{\text {Jupiter }}$, where the lower limit to $T_{\text {eff }}, \log g$ and mass correspond to the youngest age (Saumon et al. 2006). Similar analysis shows that the age of HD $3651 \mathrm{~B}$ is $3-12 \mathrm{Gyr}$, with $T_{\text {eff }}=780-840 \mathrm{~K}, \log g=5.1-5.5$ and mass $40-72 \mathrm{M}_{\text {Jupiter }}$, where the younger age gives lower $T_{\text {eff }}, \log g$ and mass (LLC). However, LLC show that the age of the HD 3651 system relative to the Gl 570 system can also be determined. The $\log \left(R_{H K}^{\prime}\right)$ chromospheric activity parameters suggest that the HD 3651 system is about five times older than the Gl 570 system; the X-ray luminosities suggest that the HD 3651 system is about twice as old as the Gl 570 system; and isochrone analyses suggest that the HD 3651 system is at least 2.5 times older than the Gl 570 system. Hence if the age of Gl 570D is $3-5 \mathrm{Gyr}$, then the age of HD 3651B is $8-12 \mathrm{Gyr}$ and the dwarf has $T_{\text {eff }}=825-840, \log g=5.4-5.5$ and mass $60-72 \mathrm{M}_{\text {Jupiter }}$ (Figure 3 of LLC).

A higher value of $\log g$ for HD 3651B than Gl 570D would seem discrepant with the observed brighter $K$-band flux peak of HD 3651B (Figure 1), as a higher gravity leads to stronger $K$-band $\mathrm{H}_{2}$ absorption at this wavelength ( $\S 3$ ). Because HD 3651B is brighter than Gl 570D at $K$, Burgasser (2007), applying the BBK technique to derive gravity, determines a lower gravity for HD $3651 \mathrm{~B}$ of $\log g=4.7$, if the HD 3651 system has solar metallicity. However, using atmospheric models, Burgasser shows that increasing metallicity results in a higher indices-implied gravity, and he derives values that agree with the luminosity- and age-driven LLC values if the system has $[\mathrm{m} / \mathrm{H}]=+0.24$.

\subsection{MASS0415-09, 2MASS0939-24 and 2MASS1114-26}

Figure 2 shows previously published low-resolution near-infrared spectra for the T8 dwarfs 2MASS0415-09 and 2MASS0939-24, and the T7.5 dwarf 2MASS1114-26. It can be seen that the spectra are similar, but 2MASS0939-24 and 2MASS1114-26 have broader $1.0 \mu \mathrm{m} Y$-peaks and are fainter at $K$ than 2MASS0415-09.

These three dwarfs were all discovered as isolated field $\mathrm{T}$ dwarfs in the 2MASS survey (Burgasser et al. 2002, Tinney et al. 2005). Their ages cannot be well constrained; however, the measured or estimated tangential velocities (see Table 1) suggest that they are not likely to be either very young, or members of the halo (see for example the discussion of kinematics in the solar region by Eggen (1998), and references therein). Their kinematics suggest ages of 1 - 10 Gyr. Evolutionary models, constrained by observed luminosity and comparisons 
with model spectra, show that the age of 2MASS0415-09 is $3-10 \mathrm{Gyr}$, with $T_{\text {eff }}=725-$ $775 \mathrm{~K}$ and $\log g=5.00-5.37$, where the younger age corresponds to lower $T_{\text {eff }}$ and $\log g$ (Saumon et al. 2007). Metallicity could not be well constrained by Saumon et al. as both $[\mathrm{m} / \mathrm{H}]=0$ and +0.3 models gave good fits to the data.

The spectral classification indices for all three of these dwarfs are very similar (Burgasser et al. 2006a), despite the fact that 2MASS1114-26 is classified 0.5 of a sub-type earlier. The $\mathrm{H}_{2} \mathrm{O}-J$ - and $\mathrm{H}_{2} \mathrm{O}-\mathrm{H}$ indices are essentially identical, while the $\mathrm{CH}_{4}-J, \mathrm{CH}_{4}-H$ and $\mathrm{CH}_{4}-K$ indices place 2MASS0939-24 and 2MASS1114-26 at a very slightly earlier type than 2MASS0415-09 (see Figure 2).

\section{Model Atmospheres}

\subsection{Description of the Models}

Models of ultracool-dwarf atmospheres have been developed by members of our team (Ackerman \& Marley 2001; Marley et al. 2002; Saumon et al. 2003). For this work, a grid of models and synthetic spectra have been calculated with effective temperature $T_{\text {eff }}=600$ - $850 \mathrm{~K}$ in steps of $50 \mathrm{~K}$; gravity $g=300,1000$ and $3000 \mathrm{~m} / \mathrm{s}^{2}$ (or in the commonly-used c.g.s units, $\log g=4.5,5.0$ and 5.5); and metallicity $[\mathrm{m} / \mathrm{H}]=-0.3,0$ and +0.3 . To begin to explore lower temperatures, $500 \mathrm{~K}$ and $550 \mathrm{~K}$ solar metallicity models with $g=300$ and 1000 $\mathrm{m} / \mathrm{s}^{2}$ have also been calculated.

These models yield temperature-pressure-composition structures under conditions of radiative-convective equilibrium using the thermal radiative transfer source function technique of Toon et al. (1989). For the very low-temperature dwarfs considered here, cloud decks of iron and silicate condensates are expected to lie below the photosphere and do not impact the spectral energy distribution except by reduction of the abundances of $\mathrm{O}, \mathrm{Fe}, \mathrm{Si}$, $\mathrm{Mg}, \mathrm{Ti}$, and other refractory elements. Thus we neglect grain opacities in the atmospheric structure and spectral calculations, although grain formation is included in the calculation of the chemical equilibrium.

The chemical equilibrium calculations for the atmosphere models are described in Lodders \& Fegley (2002) and use the solar system abundances of Lodders (2003). There is debate about the solar CNO abundance values (e.g. Ayres, Plymate \& Keller 2006, Allende Prieto

2007), which strongly impact brown dwarfs' spectral energy distributions (SEDs) as they are shaped by opacities involving these species. Studies of line formation in the solar atmosphere by Asplund et al. (2004) have determined carbon, oxygen and nitrogen abundances 0.13-0.17 dex (35-50\%) lower than those previously given by Grevesse \& Sauval (1998). The 
Lodders (2003) CNO abundances are 0.07-0.12 dex higher than the Asplund et al. values, and 0.02-0.07 dex lower than the Grevesse \& Sauval (1998) values. The total mass fraction of all heavy elements is 0.015 compared to Grevesse \& Sauval's 0.017 and Asplund et al.'s 0.012 .

The gas opacity database includes the molecular lines of $\mathrm{H}_{2} \mathrm{O}, \mathrm{CH}_{4}, \mathrm{CO}, \mathrm{NH}_{3}, \mathrm{H}_{2} \mathrm{~S}$, $\mathrm{PH}_{3}, \mathrm{TiO}, \mathrm{VO}, \mathrm{CrH}, \mathrm{FeH}$ and $\mathrm{CO}_{2}$, the atomic lines of the alkali metals ( $\mathrm{Li}, \mathrm{Na}, \mathrm{K}, \mathrm{Rb}$ and $\mathrm{Cs}$ ), and continuum opacity from $\mathrm{H}_{2}$ collisionally induced absorption (CIA), $\mathrm{H}_{2}, \mathrm{H}$ and $\mathrm{He}$ Rayleigh scattering, bound-free opacity from $\mathrm{H}^{-}$and $\mathrm{H}_{2}^{+}$, and free-free opacity from $\mathrm{H}^{-}, \mathrm{He}$, $\mathrm{H}_{2}^{-}$, and $\mathrm{H}_{2}^{+}$(Freedman, Marley \& Lodders 2007). For further discussion of the opacities important in brown dwarf photospheres see Sharp \& Burrows (2007). For this work, the line list for $\mathrm{CH}_{4}$ (which does not include transitions at $\lambda<1.5 \mu \mathrm{m}$ ) has been supplemented with the Strong et al. (1993) $\mathrm{CH}_{4}$ laboratory data for 1.05-1.5 $\mu \mathrm{m}$ and the $\mathrm{CH}_{4}$ opacity derived from the spectrum of Saturn by Karkoschka (1994) below $1 \mu \mathrm{m}$. The line list for $\mathrm{NH}_{3}$ does not include wavelengths shorter than $1.4 \mu \mathrm{m}$, and is incomplete at 1.4-1.9 $\mu \mathrm{m}$. We discuss this further in $\S 7$.

The models have been further advanced to allow for the inclusion of turbulent vertical mixing, which draws up long-lived chemical species from deep, hot layers into the cool radiative photosphere, causing their observed abundances to be greater than expected for the case of chemical equilibrium. The mixing is parameterized using a diffusion coefficient

$K_{z z}$. In the turbulent convection zone we use mixing length theory to derive $K_{z z}$, but we treat it as a tunable parameter in the nominally stable radiative zone. The abundances of very stable species like $\mathrm{CO}$ and $\mathrm{N}_{2}$ are enhanced in the upper atmosphere relative to their chemical-equilibrium values, while those of $\mathrm{H}_{2} \mathrm{O}, \mathrm{CH}_{4}$ and $\mathrm{NH}_{3}$ are reduced (see e.g. discussion in Saumon et al. 2007 and references therein). For T dwarfs, non-equilibrium chemistry is very important at mid-infrared wavelengths as the abundances of $\mathrm{CO}$ and $\mathrm{NH}_{3}$ can be affected by an order of magnitude or more (e.g. Figure 3 of Saumon et al. 2006), and these species have strong features around $5 \mu \mathrm{m}$ and $10 \mu \mathrm{m}$. The effect is not as important in the near-infrared as the abundances of species producing the majority of the opacity there, $\mathrm{H}_{2} \mathrm{O}$ and $\mathrm{CH}_{4}$, are not as strongly impacted.

\subsection{Comparison of Synthetic and Observed Spectra}

Figure 3 shows synthetic spectra calculated from model atmospheres with a range of $T_{\text {eff }}, \log g$ and metallicity appropriate for the dwarfs studied here. Vertical mixing is not included in these spectra as the effects in the near-infrared are insignificant (see Figure 2 of Saumon et al. 2006). Temperature, gravity and metallicity do however impact the near- 
infrared spectral energy distribution. The $\mathrm{CH}_{4}$ and $\mathrm{H}_{2} \mathrm{O}$ absorption bands are so strong at these temperatures that the effect of changing $T_{\text {eff }}$ is quite subtle; the largest effects are calculated to occur in the blue and red wings of the $H$-band flux peak, and at the peak of the $K$-band. Increasing metallicity has a similar effect on the $K$-band to decreasing gravity, although the effect on the $Y$-band differs. The $2 \mu \mathrm{m}$ flux is very sensitive to gravity and metallicity because of the pressure-induced $\mathrm{H}_{2}$ opacity; the $1 \mu \mathrm{m}$ flux is controlled by the $\mathrm{H}_{2} \mathrm{O}$ opacity which is more sensitive to metallicity than gravity.

Modelling these atmospheres is extremely complex. While great advances have been made (and continue to be made), there are known deficiencies and uncertainties in the models. In particular, the molecular line lists for $\mathrm{CH}_{4}$ and $\mathrm{NH}_{3}$ are incomplete or even nonexistent at $\lambda \leq 1.7 \mu \mathrm{m}$ (e.g. Saumon et al. 2007). Figure 4 tests the models by comparing the synthetic and observed spectra for the two well-studied dwarfs 2MASS0415-09 and Gl 570D. Note that the synthetic spectra have not been scaled to fit, but instead the known distance and structural calculations of the radius (determined by $T_{\text {eff }}$ and gravity) are combined to determine the flux at the Earth for each dwarf. The observed luminosity and robust evolutionary calculations can constrain $T_{\text {eff }}$ and gravity for the dwarfs to a small allowed range as described in $\S 2$. The synthetic spectra in Figure 4 fall within these ranges although they may not be optimal at the 0.2 dex level for gravity and metallicity.

Figure 4 illustrates the successes and remaining failures of current models. Given the complexity of the model physics, the match to the observations is good, but there are known problems. Incompleteness in the $\mathrm{CH}_{4}$ and $\mathrm{NH}_{3}$ opacities most likely lead to the excess model flux in the $Y, J$ and $H$ bands (this is discussed further in $\S 7$ ). The $\mathrm{K}$ I doublet at $1.25 \mu \mathrm{m}$ is too strong; this is probably due to remaining errors in the temperature-pressure profile for the atmosphere, to which the condensation of $\mathrm{K} \mathrm{I}$ into $\mathrm{KCl}$ is very sensitive (e.g. Lodders 1999 Figure 2). The shortfall in flux in the $K$-band may be due to low CNO abundances (mimicking metal deficiency), or to errors in the pressure-induced $\mathrm{H}_{2}$ opacity.

Using Gl 570D and 2MASS0415-09 as templates, and understanding the remaining model deficiencies, we now study the three very-late T dwarfs, HD 3651B, 2MASS0939-24 and 2MASS1114-26 in detail. We start with HD 3651B, for which new data have been obtained.

\section{New Observations of HD 3651B}

The Gemini Near-Infrared Spectrograph (GNIRS, Elias et al. 2006) was used in crossdispersed mode with a $0^{\prime \prime} 3$ slit to obtain a $0.9-2.5 \mu \mathrm{m} R=1700$ spectrum of HD3651B on 
2006 September 23, through program GS-2006B-DD-5. The exposure time was 600 s, and HD3651B was nodded 6" along the slit in one "ABBA" pattern, for a total of 40 minutes on-source integration time. The F2V star HIP 118301 was used to remove telluric features, and flat fields and wavelength calibration were obtained using lamps in the on-telescope calibration unit. The data were reduced using IRAF and FIGARO software packages. A slit position angle of 200 degrees was used, and no contamination was seen from the bright primary $43^{\prime \prime}$ to the north-west.

Figure 5 shows the final spectrum, compared to a similar resolution spectrum from McLean et al. (2003) for Gl 570D. As in the low-resolution spectra for these dwarfs (Figure 1 ), the two spectra are very similar, and what looks like noise is in fact real structure due to, primarily, $\mathrm{H}_{2} \mathrm{O}$ and $\mathrm{CH}_{4}$ absorption. The apparent feature seen in Figure 5 for HD 3651B around $1.27 \mu \mathrm{m}$ is not seen in Figure 1 and is most likely due to incomplete removal of the strong sky lines at this wavelength. The only significant difference between HD $3651 \mathrm{~B}$ and Gl 570D appears in the $K$-band, where the absorption features of HD 3651B are stronger.

\section{Re-Examination of HD 3651B}

\subsection{Near-Infrared Spectra}

Figure 6 shows the observed low-resolution near-infrared spectrum of HD 3651B and compares it to synthetic spectra with $T_{\text {eff }}=800$ and $850 \mathrm{~K}, \log g=5.0$ and 5.5 , and metallicity $[\mathrm{m} / \mathrm{H}]=0$ and +0.3 , covering the range of values determined for this dwarf by LLC (§2.1). These spectra have been scaled to the flux at the Earth, given the known distance to the dwarf and the calculated radii appropriate for the values of $T_{\text {eff }}, g$ and $[\mathrm{m} / \mathrm{H}]$. Comparison of Figure 6 to the template model fits of Figure 4 shows that for either the $800 \mathrm{~K}$ or $850 \mathrm{~K}$ models, the $\log g=5.5$ and $[\mathrm{m} / \mathrm{H}]=0$ spectra produce too low a $K$-band flux, and hence this combination of gravity and metallicity can be excluded. An average of the $\log g=5.0$ and $5.5,[\mathrm{~m} / \mathrm{H}]=0$ and $+0.3,800 \mathrm{~K}$ spectra shown in the top panel, weighted towards the higher gravity spectrum for less flux at $1.2 \mu \mathrm{m}$, would produce a similar fit to the one seen for Gl 570D in Figure 4. However the flux at the $H$ and $K$ peaks would be low, implying a slightly hotter temperature as suggested by the $850 \mathrm{~K} \log g=5.5[\mathrm{~m} / \mathrm{H}]=+0.3$

comparison. A temperature as high as $850 \mathrm{~K}$ can be excluded by the excessive $1.60-1.75 \mu \mathrm{m}$ flux.

The bottom panel compares the observed spectrum to a synthetic spectrum for $T_{\text {eff }}=$ $825 \mathrm{~K}, \log g=5.5$ and $[\mathrm{m} / \mathrm{H}]=+0.3$ (calculated by averaging the 800 and $850 \mathrm{~K}$ spectra). The quality of the fit is indeed similar to that for Gl 570D in Figure 4, although a slightly 
lower metallicity would improve the match at $Y$, and this would then require a decrease in gravity to maintain the match at $K$. Hence our best fits have $T_{\text {eff }} \approx 825$ with $\log g=5.4-5.5$ and $[\mathrm{m} / \mathrm{H}] \approx+0.2$. This metallicity is somewhat higher than the measured values of +0.09 to +0.16 (Gray et al. 2003, Santos et al. 2004, Valenti \& Fischer 2005). However Valenti $\&$ Fischer find a range for individual elements from +0.11 for $[\mathrm{Ti} / \mathrm{H}]$ to +0.24 for $[\mathrm{Na} / \mathrm{H}]$ and so $[\mathrm{m} / \mathrm{H}] \approx+0.2$ is not unreasonable. Note that although there are uncertainties in the absolute values of the solar chemical abundances (§3.2), they do not affect the conclusion reached here that HD 3651B is 0.2 dex more metal-rich than Gl 570D, which has effectively solar chemistry $(\S 2.1)$.

Figure 7 plots the observed medium-resolution $K$-band spectra of Gl 570D and HD 3651B with the synthetic spectra for the $\left[T_{\text {eff }}, \log g,[\mathrm{~m} / \mathrm{H}]\right]$ models $[800,5.0,0.0]$ and $[800,5.5$, $+0.3]$. Although there are discrepancies between the data and the models, the relative depths of the observed and modelled features around $2.05 \mu \mathrm{m}$ and $2.14-2.18 \mu \mathrm{m}$ (see also Figure 5) supports the conclusions reached above that HD 3651B has a higher gravity and higher metallicity than Gl 570D.

\subsection{IRAC Photometry}

Spitzer IRAC mid-infrared four-channel photometry (Fazio et al. 2004) is available for both Gl 570D (Patten et al. 2006) and HD 3651B (Luhman et al. 2007). Saumon et al. (2006) show that the $6-14 \mu \mathrm{m}$ spectrum of Gl 570D obtained with the Spitzer Infrared Spectrograph (IRS, Houck et al. 2004) matches very well the synthetic spectrum generated from a model with $T_{\text {eff }}=821 \mathrm{~K}, \log g=5.23,[\mathrm{~m} / \mathrm{H}]=0.0$ and $K_{z z}=10^{2} \mathrm{~cm}^{2} \mathrm{~s}^{-1}$. As the distances to the two dwarfs are known, we can compare the IRAC absolute magnitudes calculated by the models to the observed values (note that the IRAC magnitudes cannot be literally interpreted as a flux at the nominal filter wavelength, Cushing et al. 2006).

Figure 8 plots the observed IRAC absolute magnitudes for the two dwarfs, together with modelled values for $T_{\text {eff }}=825 \mathrm{~K}$ and vertical mixing coefficients $K_{z z}=0$ (i.e. equilibrium chemistry) and $K_{z z}=10^{4}$. The modelled values have been determined by interpolating between the 800 and $850 \mathrm{~K}$ synthesized photometry. $\log g=5.25$ and $[\mathrm{m} / \mathrm{H}]=0$ values appropriate for Gl 570D are shown, as well as $\log g=5.5$ and $[\mathrm{m} / \mathrm{H}]=+0.3$ values, appropriate for HD 3651B. The agreement between observed and modelled values for both dwarfs is excellent for the non-equilibrium chemistry case, with an average deviation of 0.10 magnitudes. The deviation between the observed and modelled $K_{z z}=0$ magnitudes is on average 0.2 magnitudes for both dwarfs, with individual band discrepancies as large as 0.3 magnitudes. Hence non-equilibrium chemistry must be included in studies of the mid-infrared fluxes of 
the late T dwarfs, as also found by Golimowski et al. (2004), Patten et al. (2006) and Leggett et al. (2007).

\subsection{Adopted Parameters for HD 3651B}

Our best-fit parameters for HD 3651B are summarised in Table 2. Comparison to the well-studied dwarf Gl 570D, and synthetic spectra, show that HD 3651B has $T_{\text {eff }}=820-$ $830 \mathrm{~K}, \log g=5.4-5.5$ and $[\mathrm{m} / \mathrm{H}] \approx+0.2$. The corresponding age and mass is $8-12 \mathrm{Gyr}$ and $60-70 \mathrm{M}_{\text {Jupiter }}$ (Figure 3 of LLC). Our derived gravity agrees with the high end of the range determined by LLC (Table 1), which is consistent with an age at the high end of their allowed range. The latter is in turn consistent with the relative ages of the Gl 570 and HD 3651 systems $(\S 2.1)$. The low end of our range of values for $T_{\text {eff }}, \log g$, age and mass agree with the values derived by Burgasser (2007) when the BBK flux indices are recalibrated for a metallicity of $[\mathrm{m} / \mathrm{H}]=+0.24$, consistent with the value implied by our model fit. The

Burgasser values for lower metallicity require the HD 3651 system to be younger than $4.7 \mathrm{Gyr}$ (see his Table 1), which is inconsistent with the various age indicators described in $\S 2.1$.

\section{Re-examination of 2MASS0939-24 and 2MASS1114-26}

The distances to 2MASS0939-24 and 2MASS1114-26 have not yet been published and so an absolute comparison of synthetic to observed spectra, i.e. with a known scale factor similar to Figures 4 and 6, cannot be made for these dwarfs. Neither can their luminosities be measured and $T_{\text {eff }}$ derived from structural models, as has been done for 2MASS0415-09, Gl 570D and HD 3651B. Nevertheless, a comparison of the model spectra shown in Figure 3 to the observed spectra shown in Figure 2 allows us to constrain $T_{\text {eff }}, \log g$ and $[\mathrm{m} / \mathrm{H}]$ for them, relative to the well-studied dwarf 2MASS0415-09. As described in $\S 2$, Saumon et al. (2007) shows that the age of 2MASS0415-09 is $3-10 \mathrm{Gyr}$, with $T_{\mathrm{eff}}=725-775 \mathrm{~K}$ and $\log g=5.00-5.37$; metallicity could not be constrained. We suggest that 2MASS0415-09 has solar or only slightly higher metallicity, as Figure 4 shows that the quality of the fit, especially in the metallicity-sensitive $K$-band, is very similar to that of $\mathrm{Gl} 570 \mathrm{D}$, for which a solar metallicity has been measured $(\S 2.1)$.

Figure 3 shows that the ratio of the $H$-band flux peak to the depth of the $\mathrm{CH}_{4}$ absorption around $1.7 \mu \mathrm{m}$, is sensitive to $T_{\text {eff }}$. Although the model opacities are incomplete in this region, we assume the strength of this absorption can still be used for relative estimates of $T_{\text {eff. }}$ Hence $T_{\text {eff }}$ for 2MASS0939-24 and 2MASS1114-26 appears to be essentially identical to 
that for 2MASS0415-09, as would also be surmised from the strengths of the $\mathrm{H}_{2} \mathrm{O}$ absorptions at 1.15 and $1.45 \mu \mathrm{m}$.

The suppressed $K$-band flux for 2MASS0939-24 and 2MASS1114-26, relative to 2MASS041509, can be explained by either higher gravity or lower metallicity. These effects however can be distinguished by their effects on the $Y$-band, as shown in Figure 3. (Again, although the models do not perfectly reproduce the $Y$-band observation, we believe that comparisons in a relative sense are valid.) The relative heights of the $K$-bands and the width of the $Y$-band flux peaks suggest that both 2MASS0939-24 and 2MASS1114-26 are quite metal-poor compared to 2MASS0415-09. Adopting solar metallicity for 2MASS0415-09 implies $[\mathrm{m} / \mathrm{H}] \approx-0.3$ for these two dwarfs. The additional suppression at $K$ for 2MASS0939-24 suggests that it also has a higher gravity than 2MASS0415-09.

Our adopted parameter ranges for these dwarfs are given in Table 2 and are: $T_{\text {eff }}=$ $725-775 \mathrm{~K},[\mathrm{~m} / \mathrm{H}] \approx-0.3$, and $\log g \approx 5.0-5.3$ for $2 \mathrm{MASS} 1114-26$ and $5.3-5.45$ for 2MASS0939-24. The upper gravity limit for 2MASS0939-24 is determined by evolutionary arguments, assuming an upper age limit of 12 Gyr for the dwarf. We do not confirm the lower temperature of $\lesssim 700 \mathrm{~K}$ determined for these dwarfs by BBK (Table 1 ); instead we find that these dwarfs each have $T_{\text {eff }}$ essentially identical to that of 2MASS0415-09, and suggest that the method of BBK needs to be revised to take metallicity into account (as done for HD 3651B by Burgasser 2007).

Saumon et al. (2007) and LLC show that $T_{\text {eff }}$ and gravity give mass and age for a brown dwarf, and that changing metallicity by 0.3 dex impacts derived mass and age by $<5 \%$. We derive ages and masses for these metal-poor late- $\mathrm{T}$ dwarfs of $10-12$ Gyr and $50-65 \mathrm{M}_{\text {Jupiter }}$ for 2MASS0939-24, and 3-8 Gyr and 30 - 50 $\mathrm{M}_{\text {Jupiter }}$ for 2MASS1114-26. When available, parallaxes will constrain these parameters further.

\section{Dwarfs Cooler than $700 \mathrm{~K}$ and the Next Spectral Type}

\subsection{Expected Spectral Trends and the Opacity Problem}

Many researchers are currently searching for objects cooler than the coldest known brown dwarfs with $T_{\text {eff }} \approx 700 \mathrm{~K}$, and in particular for the next, cooler, spectral type, for which the letter "Y" has been suggested (e.g. Kirkpatrick 2005). Until examples of cooler dwarfs are found one cannot be certain what spectral change will trigger the use of a new letter; if no obvious spectral changes are seen, the $\mathrm{T}$ classification will need to be extended beyond $\mathrm{T} 8$. 
For practical reasons, the spectral indicators for classification of the extreme- $\mathrm{T}$ or $\mathrm{Y}$ dwarfs should remain in the far-red or near-infrared, and should be identifiable based on low spectral-resolution data, as these dwarfs will be faint. As the temperature decreases more flux emerges at mid-infrared wavelengths, relative to the red and near-infrared; the calculated ratios of the $4.5 \mu \mathrm{m}$ and $10.0 \mu \mathrm{m}$ fluxes relative to the $J$-band $1.27 \mu \mathrm{m}$ flux, in Jy, are 2.3 and 1.2 at $600 \mathrm{~K}$, and 3.4 and 1.7 at $500 \mathrm{~K}$. However, detector sensitivity in the mid-infrared is worse than in the near-infrared, such that, for example, it would take 2-5 times longer to obtain a $5 \mu \mathrm{m}$ Spitzer spectrum than a $J$-band spectrum at the same $\mathrm{S} / \mathrm{N}$ ratio with current instrumentation on $8 \mathrm{~m}$-class telescopes. Although JWST is expected to be very sensitive in the mid-infrared, it will be $\sim 6$ magnitudes more sensitive in the near-infrared.

In this Section we use planetary and laboratory data, and new model calculations, to investigate spectral trends in the near-infrared as $T_{\text {eff }}$ drops below $700 \mathrm{~K}$. If the changes are too subtle to trigger a new spectral type, it will still be important to find and understand indicators of lower temperature in the $1.0-2.2 \mu \mathrm{m}$ region, as very little flux remains in the $\mathrm{H}_{2} \mathrm{O}$ and $\mathrm{CH}_{4}$ absorption features currently used to classify the $\mathrm{T}$ dwarfs (see Figures 2 and $3)$.

A summary of the expected spectral changes at $T_{\text {eff }}<700 \mathrm{~K}$ is given by Burrows, Sudarsky \& Lunine (2003). They describe how, around $T_{\text {eff }}=600 \mathrm{~K}$, the strong red and near-infrared absorption features of $\mathrm{K} \mathrm{I}$ weaken as this element forms grains of $\mathrm{KCl}$, and $\mathrm{NH}_{3}$ features become apparent in the blue wings of the $H$ and $K$ flux peaks, around 1.5 and $1.95 \mu \mathrm{m}$. Below $400 \mathrm{~K}$, water clouds are expected to form causing the colors of dwarfs to change dramatically. It is possible that the $\mathrm{NH}_{3}$ features in the $H$ - and $K$-bands may trigger the next spectral type. $\left(\mathrm{NH}_{3}\right.$ is already seen at $10 \mu \mathrm{m}$ in $\mathrm{T}$ dwarfs (Roellig et al. 2004), however its appearance in the more easily measured near-infrared could be used as a trigger in the same way that $\mathrm{CH}_{4}$ at $H$ and $K$ is used to trigger the $\mathrm{T}$ type, despite $\mathrm{CH}_{4}$ absorption being prominent at $3 \mu \mathrm{m}$ in mid- and late-L dwarfs (Noll et al. 2000)). However, although our $700 \mathrm{~K}$ and $600 \mathrm{~K}$ model spectra in Figure 3 (and our $500 \mathrm{~K}$ spectra, not shown) show a change in the shape of the $Y$-band flux peak, and a steady decline of the $K$-band flux, there is no obvious spectral change that would indicate the necessity of a new spectral type.

Unfortunately, both our analysis and that of Burrows, Sudarsky \& Lunine, suffer from incomplete or non-existent line lists for the important opacity sources $\mathrm{CH}_{4}$ and $\mathrm{NH}_{3}$. The models shown here use line lists for $\mathrm{CH}_{4}$ at $\lambda>1.5 \mu \mathrm{m}$, and $\mathrm{CH}_{4}$ laboratory and astrophysical data (Strong et al. 1993; Karkoschka 1994) for $\lambda \leq 1.5 \mu \mathrm{m}$. For $\mathrm{NH}_{3}$, a line list is used for $\lambda>1.4 \mu \mathrm{m}$, but it is known to be incomplete at $1.4-1.9 \mu \mathrm{m}$ and no $\mathrm{NH}_{3}$ opacity is included at shorter wavelengths. Discrepancies between synthetic spectra and the observations can 
be seen in the $Y$-, $J$ - and $H$-bands in Figure 4, most likely due to these incomplete opacities. Due to these problems, we revisit the predictions of the SEDs of dwarfs cooler than $700 \mathrm{~K}$, first from the observational and then from the theoretical point of view.

\subsection{Observational Indicators}

Figure 9 shows observed $0.96-2.2 \mu \mathrm{m}$ spectra of the $750 \mathrm{~K}$ dwarf 2MASS0415-09, the $\sim 160 \mathrm{~K}$ planet Jupiter ${ }^{1}$, and the $\sim 95 \mathrm{~K}$ planet Saturn. Also shown are room-temperature laboratory data for $\mathrm{CH}_{4}$ (Cruikshank \& Binder 1968) and $\mathrm{NH}_{3}$ (Irwin et al. 1999). The physical environment of the brown dwarf atmosphere will be quite different from either the laboratory gas or the planets' atmospheres. Also, there is no $\mathrm{H}_{2} \mathrm{O}$ in the planets' spectrum as all $\mathrm{H}_{2} \mathrm{O}$ is condensed in deep cloud decks. Moreover, the planetary spectra are reflection spectra. However, both planets have $\mathrm{NH}_{3}$ features in their spectra, with the warmer Jupiter having less condensed $\mathrm{NH}_{3}$ and stronger $\mathrm{NH}_{3}$ gas absorption. Given the absence of more appropriate data, we use these spectra to investigate what changes might occur at cold temperatures.

Starting at the longest wavelength, it can be seen that there is very little flux from Saturn or Jupiter in the $K$-band, due to strong pressure-induced $\mathrm{H}_{2}$ opacity. Figure 3 shows that brown dwarfs also become very faint at $K$ with decreasing temperature. Figure 9 shows that the observed T8 $\mathrm{K}$-band spectrum is very complex, due to the wealth of $\mathrm{H}_{2} \mathrm{O}$ and $\mathrm{CH}_{4}$, and possibly $\mathrm{NH}_{3}$, absorption features (see also the higher resolution spectra of the T7.5 dwarfs in Figures 5 and 7, and the discussion of the Gl 229B K-band spectrum in Saumon et al. 2000). The feature seen in 2MASS0415-09 at $2.02 \mu \mathrm{m}$ is as strong in hotter $800 \mathrm{~K}$ spectra (Figures 1 and 3) and so is not a useful spectral indicator despite the apparent match to an $\mathrm{NH}_{3}$ feature in the laboratory spectrum. Although the blue wing of this band was suggested by Burrows et al. (2003) as a region where increasing $\mathrm{NH}_{3}$ absorption would become apparent for the temperature range of interest, Figures 3 and 9 show that identifying the increasing $\mathrm{NH}_{3}$ opacity at $1.95-2.05 \mu \mathrm{m}$ amongst the existing features, and with low flux levels, is not practical. Hence the $K$-band is not an appropriate region to search for features that may indicate the next spectral type.

The $H$-band offers only a very narrow region of peak flux due to the strong $\mathrm{H}_{2} \mathrm{O}$ features to the blue, and the strong $\mathrm{CH}_{4}$ to the red of this band. Identifying the increasing $\mathrm{NH}_{3}$ opacity at $1.48-1.55 \mu \mathrm{m}$, as suggested by Burrows et al. (2003), again is problematical due

\footnotetext{
${ }^{1}$ from the IRTF Spectral Library, accessible at http://irtfweb.ifa.hawaii.edu/ spex/spexlibrary/IRTFlibrary.html see Rayner, Cushing \& Vacca (2008)
} 
to the already strong $\mathrm{H}_{2} \mathrm{O}$ absorption at these wavelengths. A steepening of the blue wing of the $H$-band peak may become visible, and we discuss this further below. If the dip seen in the laboratory spectrum of $\mathrm{NH}_{3}$ at $1.58 \mu \mathrm{m}$, which appears to carry through in the Jupiter spectrum, is also seen in brown dwarf spectra, it may be a useful $\mathrm{Y}$ dwarf indicator, as it is in a region relatively free of $\mathrm{H}_{2} \mathrm{O}$ and $\mathrm{CH}_{4}$ absorption.

It appears that the $Y$ and $J$ regions may provide the best $\mathrm{Y}$ dwarf indicators. This would be fortuitous, not only for the coincidence of the letter "Y", but because these extreme dwarfs emit more energy at $1.0-1.3 \mu \mathrm{m}$ than at $1.5-2.2 \mu \mathrm{m}$. Weak absorption features in the 2MASS0415-09 spectrum at 1.21-1.22 $\mu \mathrm{m}$ and $1.29 \mu \mathrm{m}$ apparently align with $\mathrm{NH}_{3}$ features, and are weaker or non-existent in the warmer Gl 570D spectrum. Even if these are due to $\mathrm{CH}_{4}$ and not $\mathrm{NH}_{3}$, if they become dominant features of colder dwarfs they could be used as spectral type indicators. The feature at $1.27 \mu \mathrm{m}$ is seen in the warmer spectrum and coincides with a strong sky emission feature, and so is not a useful spectral indicator.

The blue wing of the $Y$-band peak may be a useful $\mathrm{Y}$ dwarf indicator due to the loss of gaseous K I from the photosphere, as the strong $0.77 \mu \mathrm{m} \mathrm{K} \mathrm{I} \mathrm{feature} \mathrm{impacts} \mathrm{the} \mathrm{blue} \mathrm{edge}$ of this band. Also, the Jovian spectrum suggests that the $1.02-1.035 \mu \mathrm{m}$ region may show $\mathrm{NH}_{3}$ features. The feature seen in 2MASS0415-09 at $1.02 \mu \mathrm{m}$ is weak or non-existent in the Gl 570D spectrum, and so, whether due to $\mathrm{NH}_{3}$ or $\mathrm{CH}_{4}$, may be a useful later- $\mathrm{T}$ or $\mathrm{Y}$ dwarf indicator.

\subsection{Theoretical Indicators}

To further explore the appearance of $\mathrm{NH}_{3}$ in very-late $\mathrm{T}$ dwarf spectra, we have complemented our $\mathrm{NH}_{3}$ line opacity in the models with the Irwin et al. (1999) laboratory measurements, which were conducted at $T=230-296 \mathrm{~K}$ at a resolution of $5 \mathrm{~cm}^{-1}$. We applied their opacities over the range $0.91-1.9 \mu \mathrm{m}$ by extrapolating the tabulated opacities to higher temperatures using their estimate for the energy of a representative lower level for the transitions in each frequency range. As a test of the temperature extrapolation, we computed spectra with the measured opacities at a fixed temperature of $300 \mathrm{~K}$ and obtained very similar synthetic spectra.

Figure 10 shows the resulting spectra for brown dwarfs with $T_{\text {eff }}$ of 600 and $700 \mathrm{~K}$. A comparison of the $700 \mathrm{~K}$ spectrum with one computed without the Irwin et al. (1999) $\mathrm{NH}_{3}$ opacity shows that a very strong feature would be expected on the blue side of the $H$ band peak $(\sim 1.54 \mu \mathrm{m})$, that the $J$ band peak would be shaved on both sides, and that the blue side of the $Y$ band peak would be suppressed by an $\mathrm{NH}_{3}$ band. While this would 
seem a promising way of identifying $\mathrm{NH}_{3}$ features to define the spectral characteristics of $\mathrm{Y}$ dwarfs, none of these features are apparent in the spectra of the coolest known $\mathrm{T}$ dwarfs with $T_{\text {eff }} \sim 700-800 \mathrm{~K}$ (Figure 2 ). The shoulders seen in the red wing of the $J$-band and the blue wing of the $H$-band are in particular discord with the observational data (e.g. for 2MASS0415-09 in Figures 2, 4 and 9).

On the other hand, detailed analyses of cool $\mathrm{T}$ dwarfs have revealed a depletion of $\mathrm{NH}_{3}$ in the atmosphere by a factor of $\sim 10$ due to non-equilibrium chemistry arising from vertical transport in the atmosphere (Saumon et al. 2006, 2007). Figure 10 shows a $600 \mathrm{~K}$ spectrum with the Irwin et al. (1999) $\mathrm{NH}_{3}$ opacity computed out of chemical equilibrium with an eddy diffusion coefficient of $K_{z z}=10^{4} \mathrm{~cm}^{2} \mathrm{~s}^{-1}$ (see e.g. Saumon et al. 2006). The $\mathrm{NH}_{3}$ features from the Irwin et al. (1999) opacity are significantly weaker but remain stronger than seen in the observational data. It is highly unlikely that non-equilibrium chemistry can further decrease the $\mathrm{NH}_{3}$ abundance by the required factor. Additional laboratory work on the opacity of $\mathrm{NH}_{3}$ well above room temperature, as well as ab initio line list calculations, are urgently needed to understand the spectra of the new cooler brown dwarfs that will soon be uncovered by modern large surveys.

\section{Conclusions}

We have used the well-studied T7.5 and T8 dwarfs Gl 570D and 2MASS0415-09 as templates to determine physical parameters for the very similar dwarfs HD 3651B, 2MASS0939324 and 2MASS1114-26. Synthetic spectra and photometry, together with new and published near-infrared spectra and mid-infrared photometry, show that HD 3651B, a distant companion to an exoplanet host star, has both higher gravity and higher metallicity than Gl 570D. The derived parameters for this dwarf are: $T_{\text {eff }}=820-830 \mathrm{~K}, \log g=5.4-5.5,[\mathrm{~m} / \mathrm{H}]=+0.2$ and $K_{z z}=10^{4} \mathrm{~cm}^{2} \mathrm{~s}^{-1}$. The corresponding age is around $10 \mathrm{Gyr}$ and mass $65 \mathrm{M}_{\text {Jupiter }}$. Synthetic spectra, together with published near-infrared spectra, show that 2MASS0939-24 and 2MASS1114-26 are both metal-poor compared to 2MASS0415-09, and have similar or slightly higher gravities. The derived parameters for these dwarfs are: $T_{\text {eff }}=725-775 \mathrm{~K}$ and $[\mathrm{m} / \mathrm{H}]=-0.3 ; \log g=5.3-5.45$ for 2MASS0939-24 and $\log g=5.0-5.3$ for 2MASS1114-26. The corresponding ages and masses are $\sim 10 \mathrm{Gyr}$ and $60 \mathrm{M}_{\text {Jupiter }}$ for 2MASS0939-24, and 5 Gyr and $40 \mathrm{M}_{\text {Jupiter }}$ for 2MASS1114-26. These temperatures are significantly higher than

those derived by BBK, and hence these objects are not the coolest known $\mathrm{T}$ dwarfs. For these two dwarfs (which do not have published parallax values) the atmospheric properties are mostly derived from the ratio of the $J$ and $K$ flux peaks, which is sensitive to both gravity and metallicity, and the shape of the $Y$ flux peak, which is sensitive primarily to 
metallicity.

The intrinsic accuracy of the inferred atmospheric parameters depends, of course, on the veracity of the atmosphere models. Until other model sets are similarly applied to comparable datasets, the systematic uncertainties are difficult to evaluate. However, given the apparent simplicity of late $\mathrm{T}$ dwarfs (relative to the cloudy $\mathrm{L}$ dwarfs, for example), large systematic errors are unlikely. Errors larger than 5 to $10 \%$ in effective temperature and 0.3 dex in $\log g$ and metallicity would be surprising. The uncertainties here in $\log g$ and metallicity are smaller than this and are estimated to be 0.1 dex (or 26\%), as we have carried out relative comparisons to the well-studied dwarfs Gl 570D and 2MASS0415-09.

When the metallicities and gravities of more $\mathrm{T}$ dwarfs have been determined (following improvements to current models), the community will need to agree on metallicity and gravity labels in spectral classification schemes. We suggest that these labels should follow existing stellar schemes. Metal-poor main-sequence M dwarfs are labelled as "subdwarfs" due to their location in optical color-magnitude diagrams (e.g. Figure 10 of Monet et al. 1992). Although the location of brown dwarfs in such diagrams will be a strong function of gravity, the "sd" prefix could be maintained as a metal-poor indicator, as already being done for some L dwarfs (e.g. Burgasser 2004). Similarly, gravities should be described numerically, using the $\log$ of the value in c.g.s. units, following the spectral type. It would be desirable to specify gravity to at least one-half a dex, as this parameter has a large impact on brown dwarf SEDs. Also, for a given $T_{\text {eff }}$, a range of 1.0 dex in $\log g$ implies a large range in age and mass (for example if $T_{\text {eff }}=800 \mathrm{~K}, \log g=4.5$ corresponds to mass and age around $15 \mathrm{M}_{\text {Jupiter }}$ and $0.5 \mathrm{Gyr}$, while $\log g=5.5$ corresponds to $70 \mathrm{M}_{\text {Jupiter }}$ and $15 \mathrm{Gyr}$ ). Given this requirement, and the existing association of roman numerals with luminosity class, we suggest that arabic numerals are used for brown dwarf gravities, following a "g" to separate it from the spectral type. Thus a metal-poor T7.5 dwarf with $\log g=5.5$ would be described as sdT7.5g5.5. For $\mathrm{L}$ and early-T dwarfs an additional classification dimension will be required to describe the "dustiness" or "redness" of the spectrum caused by the condensate cloud decks, perhaps using the letter "r" (as "d" would be confused with "dwarf").

Existing models would greatly benefit from improved line lists for transitions of $\mathrm{CH}_{4}$ and $\mathrm{NH}_{3}$ at $\lambda \leq 1.7 \mu \mathrm{m}$. Such lists would allow substantially better analyses of the SEDs of the $\mathrm{T}$ dwarfs, and would yield better predictions for the spectra and colors of the next, cooler, spectral type. We use observed near-infrared spectra of 2MASS0415-09, Jupiter and Saturn, and laboratory observations of $\mathrm{CH}_{4}$ and $\mathrm{NH}_{3}$ gas, to investigate the appearance of this spectral type. The expectation was that the appearance of $\mathrm{NH}_{3}$ features in the blue wings of the $H$ and $K$ flux peaks would be significant enough to require a new ("Y") spectral type, and that this would occur at $T_{\text {eff }} \approx 600 \mathrm{~K}$. However, we find that the lack of flux in the 
$H$ and $K$ wings, combined with the complexity of already strong $\mathrm{H}_{2} \mathrm{O}$ and $\mathrm{CH}_{4}$ absorption features, make it unlikely that these $\mathrm{NH}_{3}$ features will be useful. Instead we find that previously overlooked $\mathrm{NH}_{3}$ features that occur in the brighter $Y$ - and $J$-bands may be the indicator of the next spectral type, combined with the changing shape of the $Y$-band as $\mathrm{K} \mathrm{I}$ solidifies into $\mathrm{KCl}$. Large uncertainties remain still, as an attempt to include $\mathrm{NH}_{3}$ laboratory data into our models produced spectra that do not agree with observations, and as it is now known from mid-infrared analyses that the abundance of $\mathrm{NH}_{3}$ in late- $\mathrm{T}$ dwarfs is around 10 times lower than expected from chemical equilibrium models. It is possible that the next spectral type may not be triggered until much cooler temperatures are reached. Progress will be made as observers continue to search to fainter limits to find cooler, and possibly spectrally different, brown dwarfs, while theoreticians continue improving the opacity line lists and models.

We are grateful for the award of Director's Discretionary time at the Gemini Observatory, which is operated by the Association of Universities for Research in Astronomy, Inc., under a cooperative agreement with the NSF on behalf of the Gemini partnership: the National Science Foundation (United States), the Particle Physics and Astronomy Research Council (United Kingdom), the National Research Council (Canada), CONICYT (Chile), the Australian Research Council (Australia), CNPq (Brazil) and CONICET (Argentina). TRG and SKL are supported by the Gemini Observatory, and acknowledge the above partnership for this support. This work was supported in part under the auspices of the U.S. Department of Energy at Los Alamos National Laboratory under Contract W-7405-ENG-36. MSM acknowledges the support of the NASA Office of Space Sciences. MCL acknowledges support for this work from NSF grants AST-0407441 and AST-0507833 and an Alfred P. Sloan Research Fellowship. Dale Cruikshank gave invaluable assistance with the $\mathrm{CH}_{4}$ and $\mathrm{NH}_{3}$ laboratory data. Katarina Lodders contributed significantly in many helpful discus-

sions. John Rayner generously provided the Jovian spectrum pre-publication. Finally, we are grateful to the referee, who significantly improved the manuscript.

\section{REFERENCES}

Ackerman, A. S. \& Marley, M. S., 2001, ApJ, 5556, 872.

Allende Prieto, C. 2007, in Proc. of the $14^{\text {th }}$ Cambridge Workshop on Cool Stars, Stellar Systems and the Sun, G. van Belle, ed. in press.

Asplund, M., Grevesse, N., Sauval, A. J., Allende Prieto, C., \& Kiselman, D., 2004, A\&A, 417,751 
Ayres, T. R., Plymate, C. \& Keller, C. U. 2006, ApJS, 165, 618

Beichman, C. A., Chester, T. J., Skrutskie, M., Low, F. J. \& Gillett, F. 1998, PASP, 110, 480

Burgasser, A. J., 2004, ApJ, 614, L73

Burgasser, A. J., 2007, ApJ, 658, 617

Burgasser, A. J. et al. 1999, ApJ, 522, L65

Burgasser, A. J. et al. 2000, ApJ, 531, L57

Burgasser, A. J. et al. 2002, ApJ, 564, 421

Burgasser, A. J., Geballe, T. R., Leggett, S. K., Kirkpatrick, J. Davy \& Golimowski, D. A. 2006a, ApJ, 637, 1067

Burgasser, A. J., Burrows, A. \& Kirkpatrick, J. D. 2006b, ApJ, 639, 1095 (BBK)

Burrows, A., Sudarsky, D. \& Lunine, J. I. 2003, ApJ, 596, 587

Cruikshank, D. P. \& Binder, A. B. 1968, Communications of the Lunar and Planetary Laboratory 6, 275

Cushing, M. C. et al. 2006, ApJ, 648, 614

Eggen O.J. 1998, AJ, 115, 2397

Elias, J. H., Rodgers, B., Joyce, R. R., Lazo, M., Doppmann, G., Winge, C., RodriguezArdila, A., 2006, in Ground-based and Airborne Instrumentation for Astronomy, eds. I. S. McLean and I. Masanori, SPIE 6269, 36

The Hipparcos and Tycho Catalogues, ESA 1997 (ESA SP-1200) (Noordwijk: ESA)

Fazio, G. et al., 2004, ApJS, 154, 10

Feltzing, S. \& Gustaffson, B. 1998, A\&AS, 129, 237

Fischer, D. A., Butler, R. P., Marcy, G. W., Vogt, S. S. \& Henry, G. W., 2003, ApJ, 590, 1081

Freedman, R.F., Marley, M.S. \& Lodders, K., 2007, ApJ, submitted

Geballe, T. R., Saumon, D., Leggett, S. K., Knapp, G. R., Marley, M. S. \& Lodders, K. 2001, ApJ, 556, 373 
Golimowski D. A. et al. 2004, AJ, 127, 3516

Gray, R. O., Corbally, C. J., Garrison, R. F., McFadden, M. T., \& Robinson, P. E. 2003, AJ, 126, 2048

Grevesse, N., \& Sauval, A. J., 1998, Space Science Reviews, 85, 161

Hewett, P. C., Warren, S. J., Leggett, S. K. \& Hodgkin, S. T. 2006, MNRAS, 367, 454

Houck, J. et al. 2004, ApJS, 154, 18

Irwin, P. G. J., Calcutt, S. B., Sihra, K., Taylor, F. W., Weir, A. L., Ballard, J. \& Johnston, W. B., 1999, JQSRT, 62, 193

Karkoschka, E. 1994, Icarus, 111, 174

Kim, S. J. and Geballe, T. R. 2005, Icarus, 179, 449,

Kirkpatrick, J. D. 2005, ARA\&A, 43, 195

Knapp, G. R. et al. 2004, AJ, 127, 3553

Lawrence, A. et al. 2007, MNRAS, in press

Leggett, S. K., Saumon, D., Marley, M. S, Geballe, T. R., Golimowski, D. A., Stephens, D. S. \& Fan, X. 2007, ApJ, 655, 1079

Liebert, J. \& Burgasser, A. J. 2007, ApJ, 655, 522

Liu, M. C., Leggett, S. K., Chiu, K. C., 2007, ApJ, 660, 1507 (LLC)

Lodders, K. 1999, ApJ, 519, 793

Lodders, K. 2003, ApJ, 591, 1220

Lodders, K. \& Fegley, B. Jr. 2002, Icarus, 155, 393

Lodieu, N. et al., 2007, MNRAS, submitted

Luhman, K. L. et al., 2007, ApJ, 654, 570

Marley, M. S., Seager, S., Saumon, D., Lodders, K., Ackerman, A. S., Freedman, R. S. \& Fan, X., 2002, ApJ, 568, 335

McLean, I. S., McGovern, M. R., Burgasser, A. J., Kirkpatrick, J. D., Prato, L. \& Kim, S. S. 2003, ApJ, 596, 561 
Monet, D. G., Dahn, C. C., Vrba, F. J., Harris, H. C., Pier, J. R., Luginbuhl, C. B. \& Ables, H. D., 1992, AJ, 103, 638

Mugrauer, M., Seifahrt, A., Neuhaeuser, R., \& Mazeh, T., 2006, MNRAS, 373, L31

Nakajima, T., Oppenheimer, B. R., Kulkarni, S. R., Golimowski, D. A., Matthews, K. \& Durrance, S. T., 1995, Nature, 378, 463

Noll, K. S., Geballe, T. R., Leggett, S. K. \& Marley, M. S., 2000, ApJ, 541, L75

Patten, B. M., et al. 2006, ApJ, 651, 502

Rayner, J. T., Cushing, M. C. \& Vacca, W. D., 2008, in preparation

Roellig, T. L. et al., 2004, ApJS, 154, 418

Santos, N. C., Israelian, G., \& Mayor, M., 2004, A\&A, 415, 1153

Santos, N. C., Israelian, G., \& Mayor, M., Bento, J. P., Almeida, P. C., Sousa, S. G., \& Ecuvillon, A., 2005, A\&A, 437, 1127

Saumon, D., Geballe, T. R., Leggett, S. K., Marley, M. S., Freedman, R. S., Lodders, K., Fegley, B. Jr. \& Sengupta, S. K., 2000, ApJ, 541, 374

Saumon, D., Marley, M. S., Lodders, K. \& Freedman, R. S., 2003, Brown Dwarfs, Proceedings of IAU Symposium 211, ed. E. Martin, San Francisco: Astronomical Society of the Pacific, 345

Saumon, D., Marley, M. S., Cushing, M. C., Leggett, S. K., Roellig, T. L., Lodders, K. \& Freedman, R. S. 2006, ApJ, 647, 552

Saumon, D. et al. 2007, ApJ, 656, 1136

Sharp, C. M. \& Burrows, A. 2007, ApJS, 168, 140

Skrutskie, M. F. et al. 2006, AJ, 131, 1163

Strong, K., Taylor, F. W., Calcutt, S. B., Remedios, J. J. \& Ballard, J. 1993, JQSRT, 50, 363

Tinney, C. G.,Burgasser, A. J. \& Kirkpatrick, J. D. 2003, AJ, 126, 975

Tinney, C. G., Burgasser, A. J., Kirkpatrick, J. D. \& McElwain, M. W. 2005, AJ, 130, 2326

Toon, O. B., McKay, C. P., Ackerman, T. P. \& Santhanam, K., 1989, JGR, 9416287 
Valenti, J. A. \& Fischer, D. A. 2005, ApJS, 159, 141

van Altena, W. F., Lee, J. T. \& Hoffleit, E. D. 1995, The General Catalogue of Trigonometric Stellar Parallaxes (4th ed.; New Haven; Yale Univ. Obs.)

Vrba, F. J. et al. 2004, AJ, 127, 2948

Warren, S. J.et al. 2007a, MNRAS, 375,213

Warren, S. J.et al. 2007b, MNRAS, submitted

York, D. G. et al. 2000, AJ, 120, 1579 


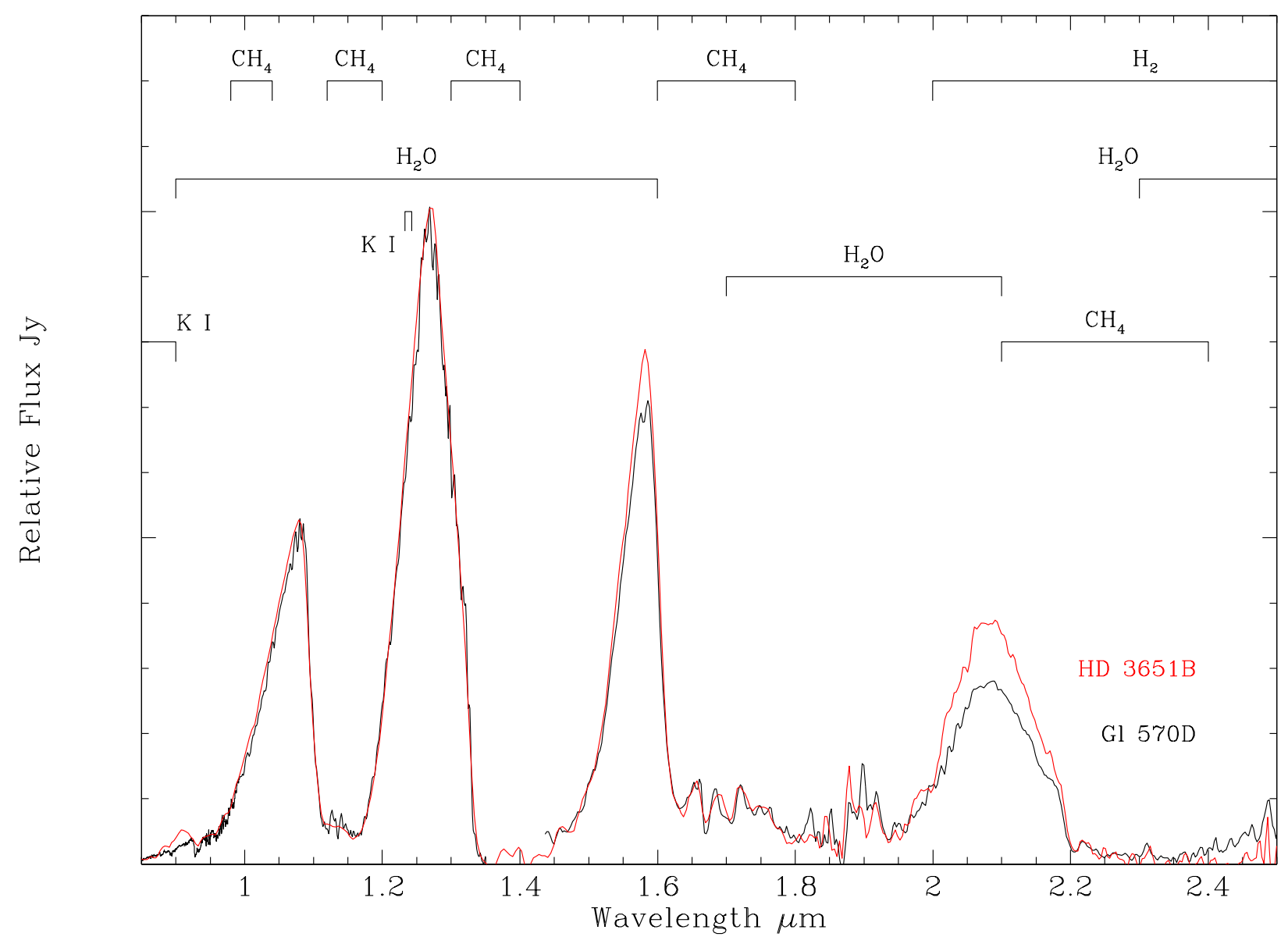

Fig. 1.- Observed R $\approx 400$ CGS4 near-infrared spectra of Gl 570D (Geballe et al. 2001) and $\mathrm{R} \approx 150$ Spex spectra of HD 3651B (Liu et al. 2007). Spectra are scaled to the peak flux at $1.27 \mu \mathrm{m}$. 


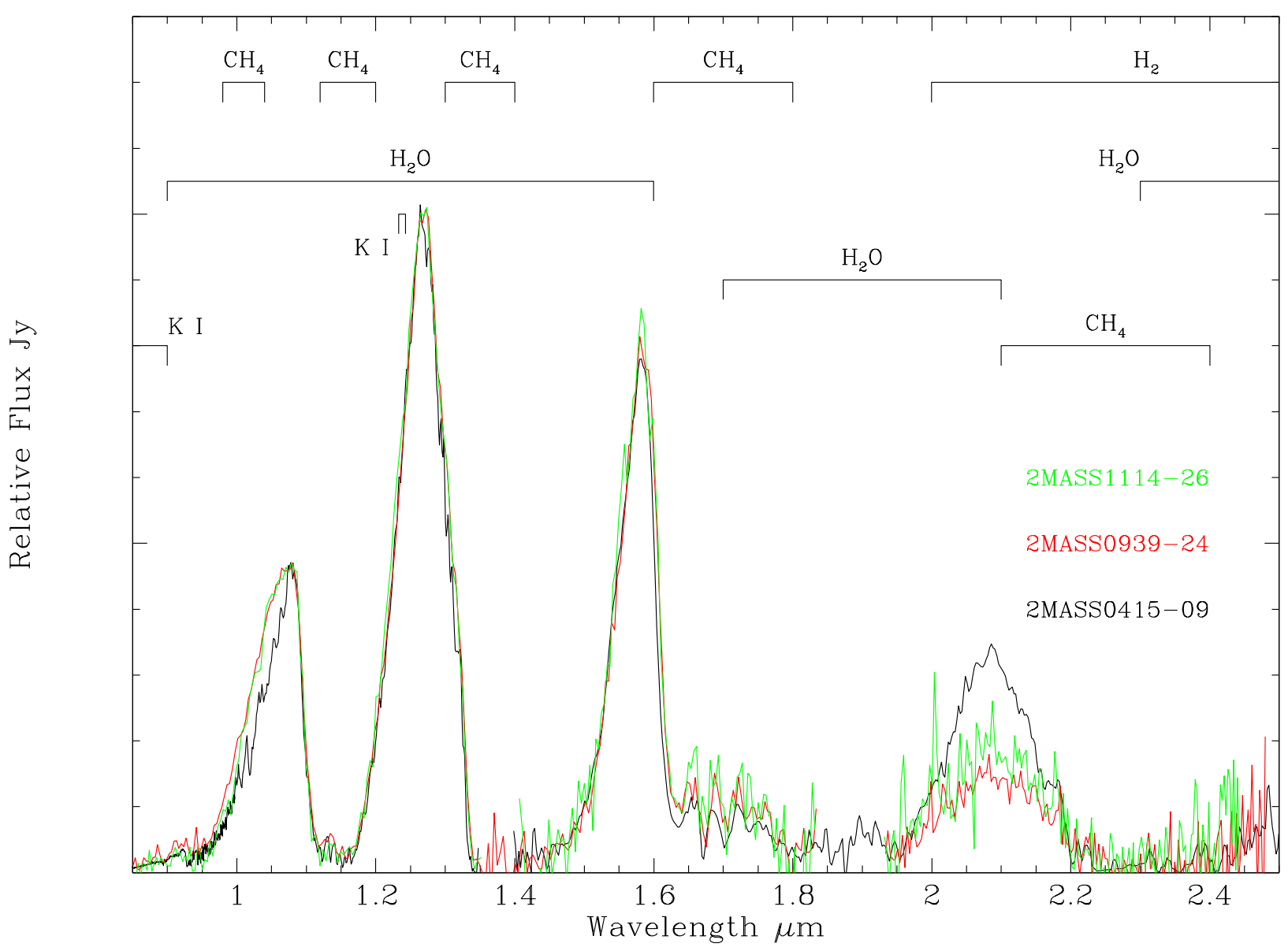

Fig. 2.- Observed $\mathrm{R} \approx 400$ CGS4 near-infrared spectra of 2MASS0415-09 (Knapp et al. 2004) and $R \approx 150$ Spex spectra of 2MASS0939-24 and 2MASS1114-26 (Burgasser, Burrows $\&$ Kirkpatrick 2006b). Spectra are scaled to the peak flux at $1.27 \mu \mathrm{m}$. 


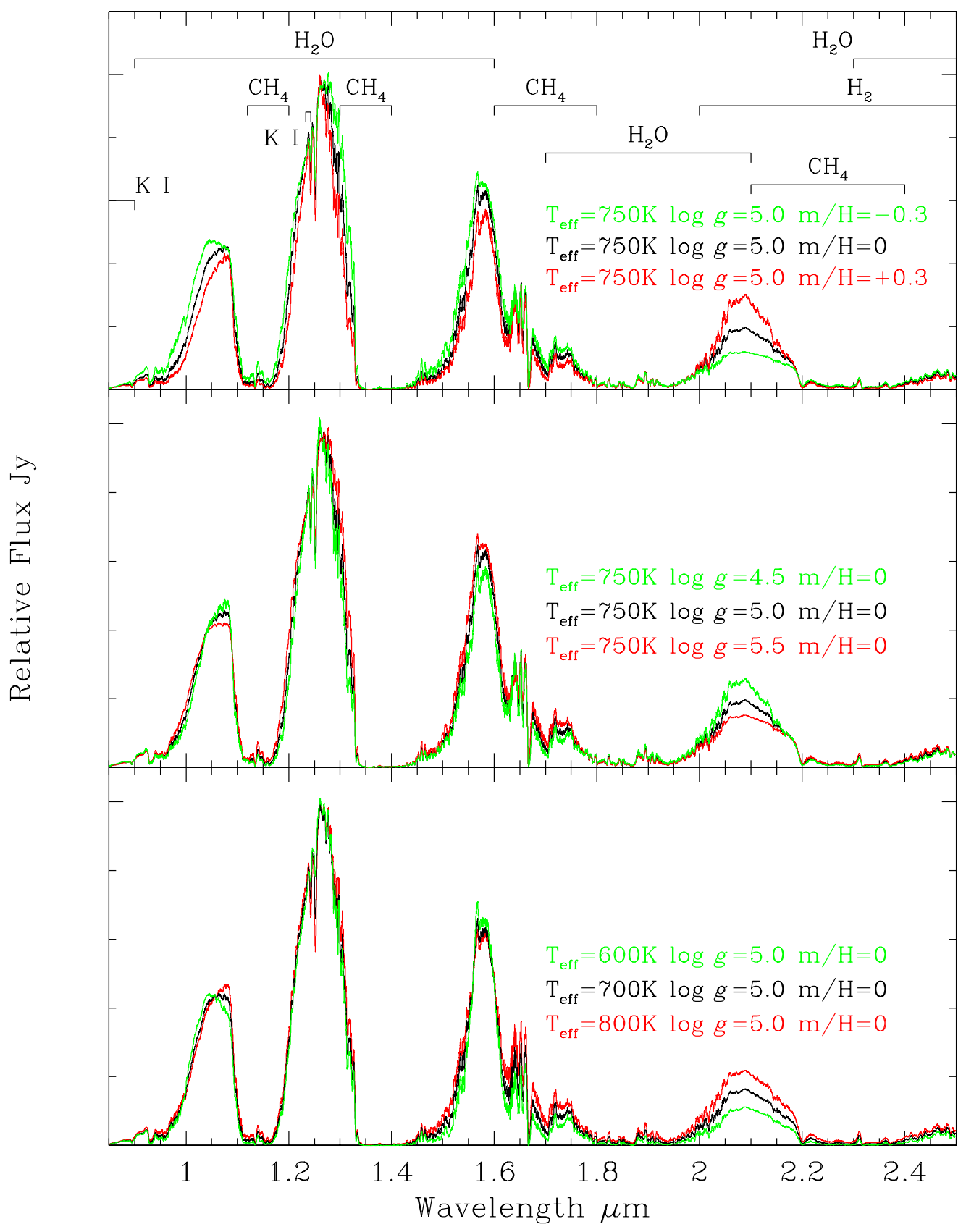

Fig. 3.- Synthetic spectra smoothed to $\mathrm{R}=400$ and scaled to the peak flux at $1.27 \mu \mathrm{m}$. 


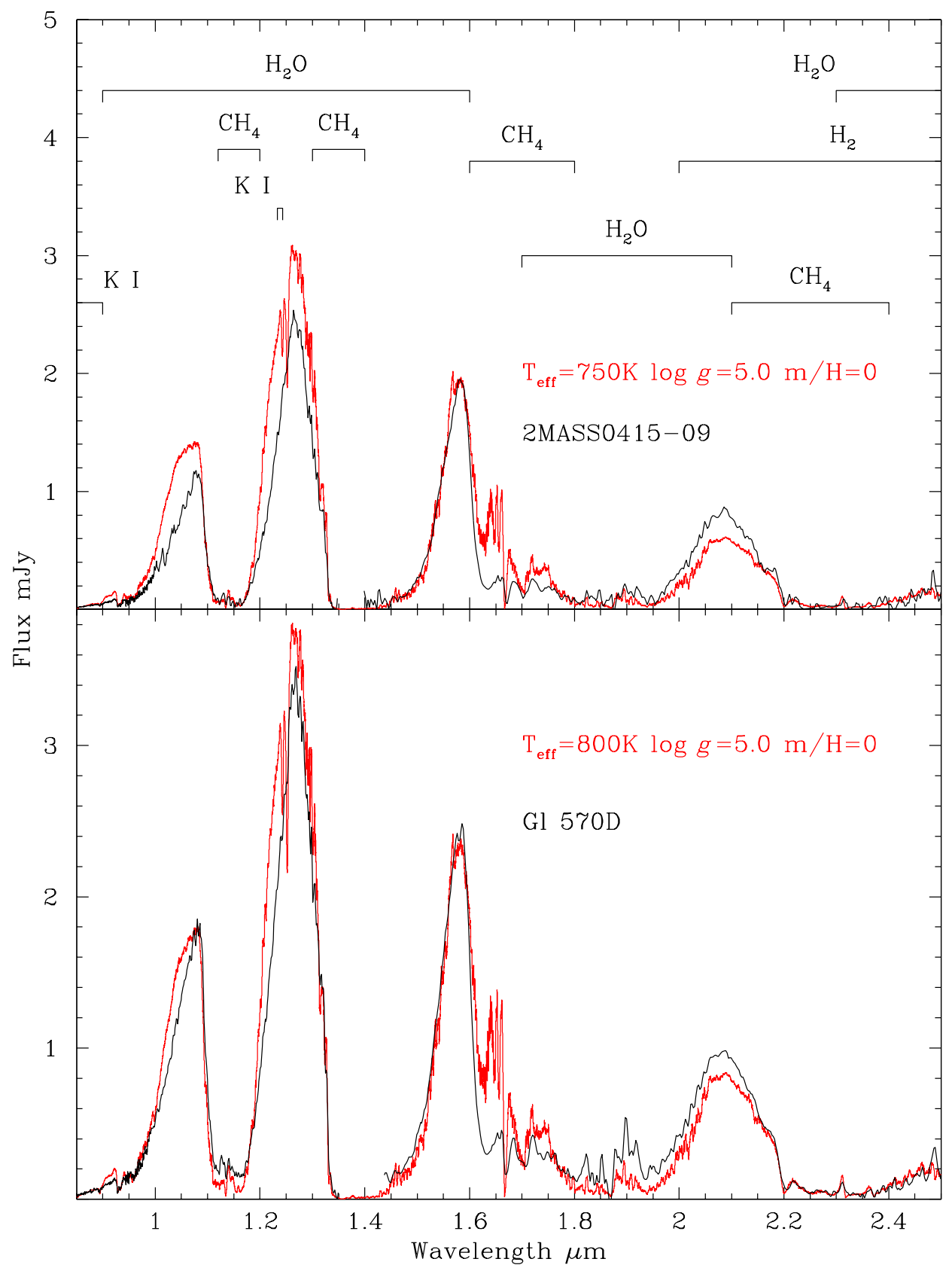

Fig. 4.- Observed $\mathrm{R} \approx 400$ near-infrared spectra of Gl 570D and 2MASS0415-09 (black), and synthetic spectra scaled for the observed flux at Earth (red). 

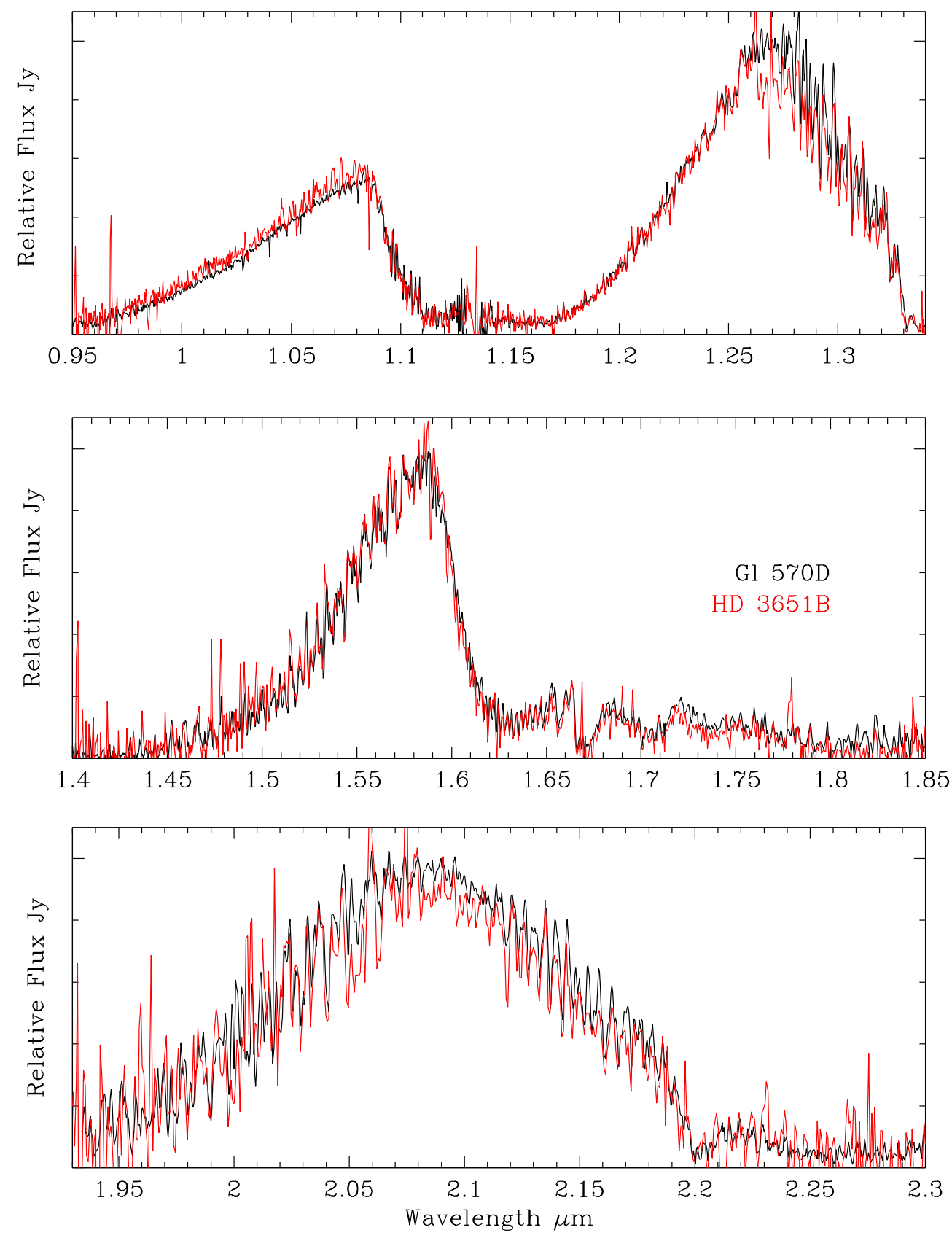

Fig. 5.- Observed medium-resolution $\mathrm{R}=1700-2000$ near-infrared spectra of Gl 570D and HD 3651B, from the NIRSPEC brown dwarf spectroscopic survey (McLean et al. 2003) and this work. Spectra are scaled to the peak flux in each panel. See Figure 1 for line identifications. 


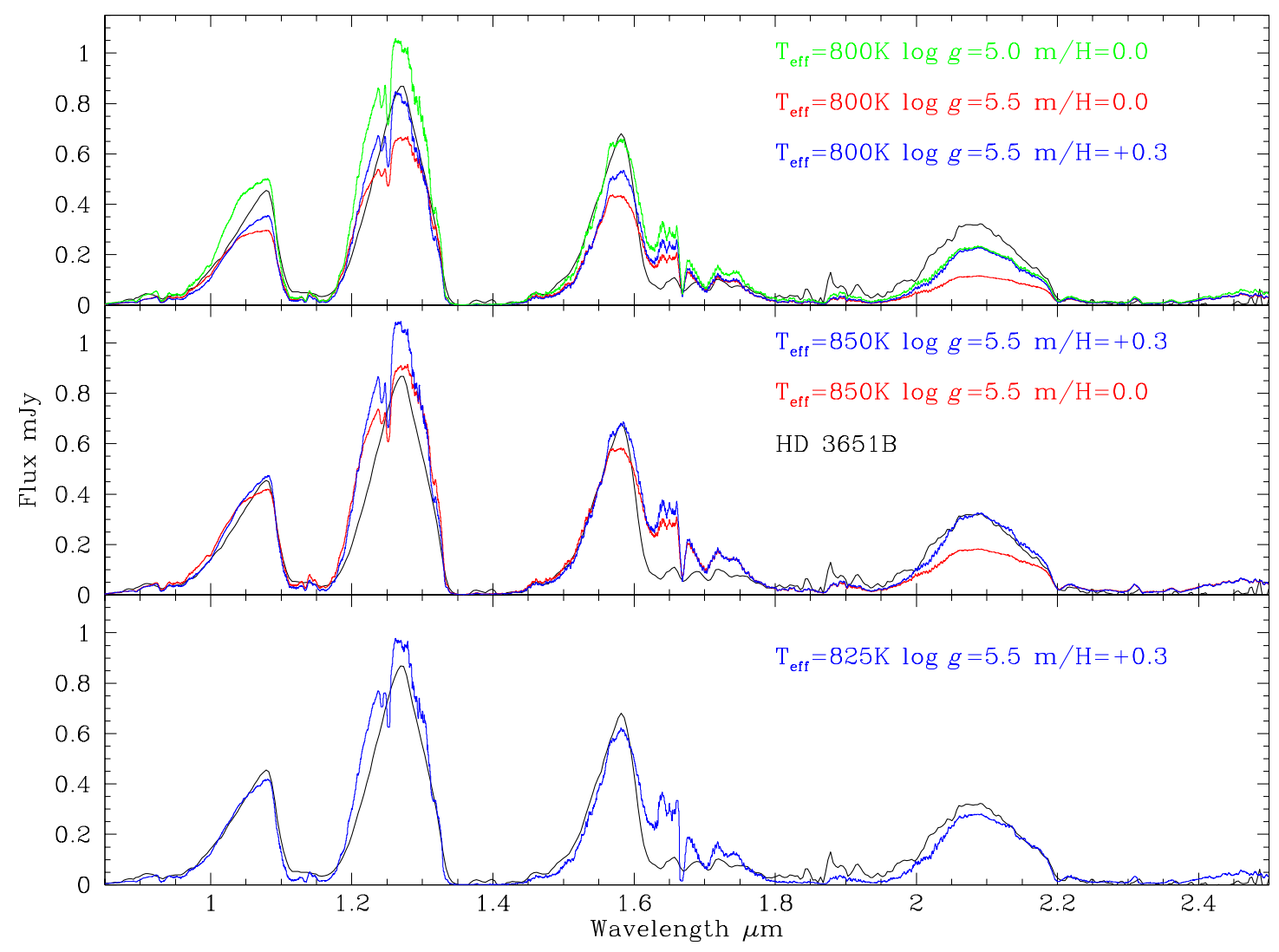

Fig. 6.- Observed low-resolution near-infrared spectrum of HD 3651B (black curve), compared to synthetic spectra scaled for the observed flux at Earth for various temperatures, gravities and metallicities. 


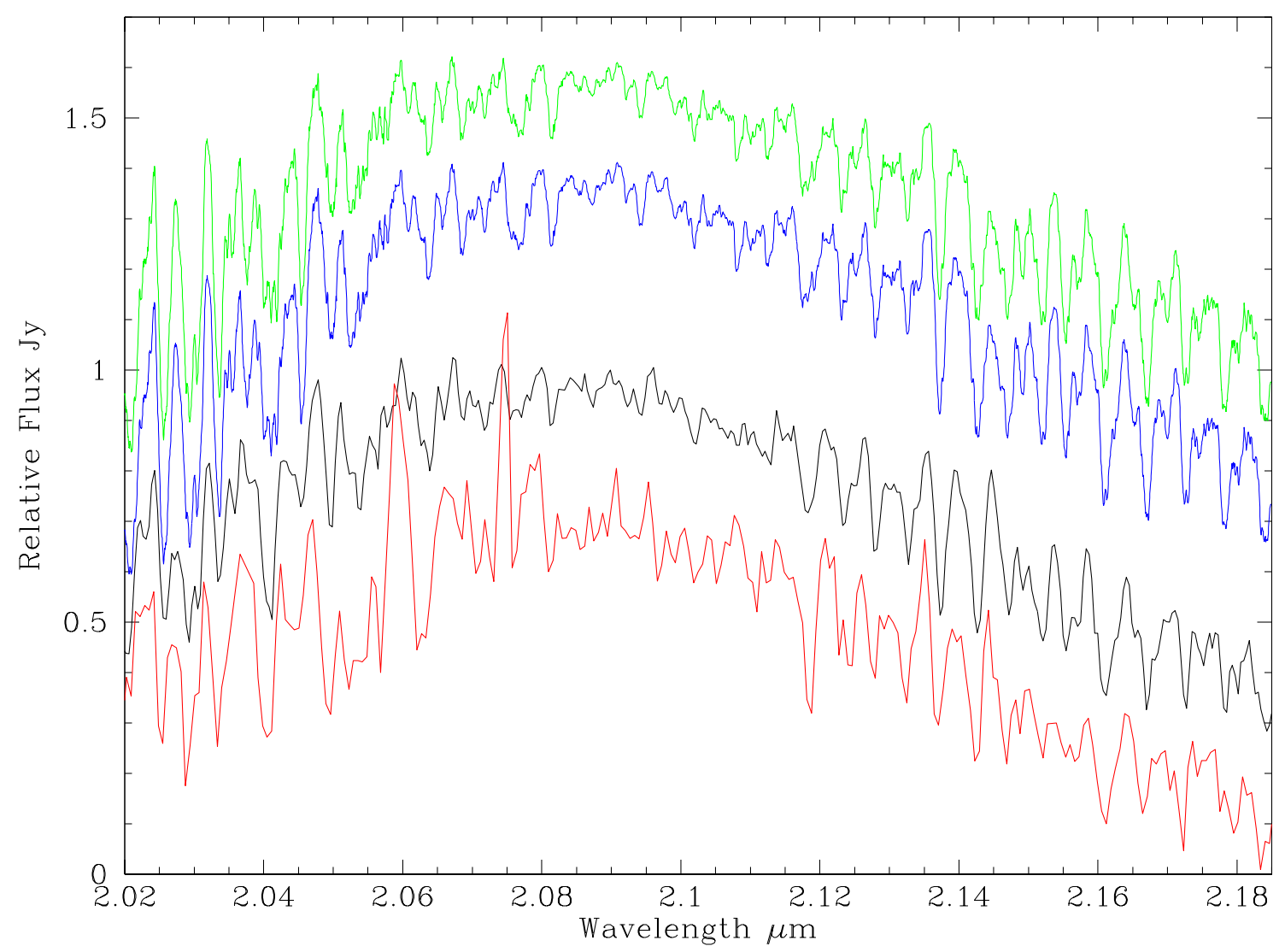

Fig. 7.- Medium-resolution spectra of Gl 570D (McLean et al. 2003, black) and HD 3651B (this work, red) compared to synthetic spectra for $\left[T_{\text {eff }}, \log g,[\mathrm{~m} / \mathrm{H}]\right]$ models $[800,5.0,0.0]$ (green) and $[800,5.5,+0.3]$ (blue). The spectra are scaled to the peak flux and offset for clarity. 


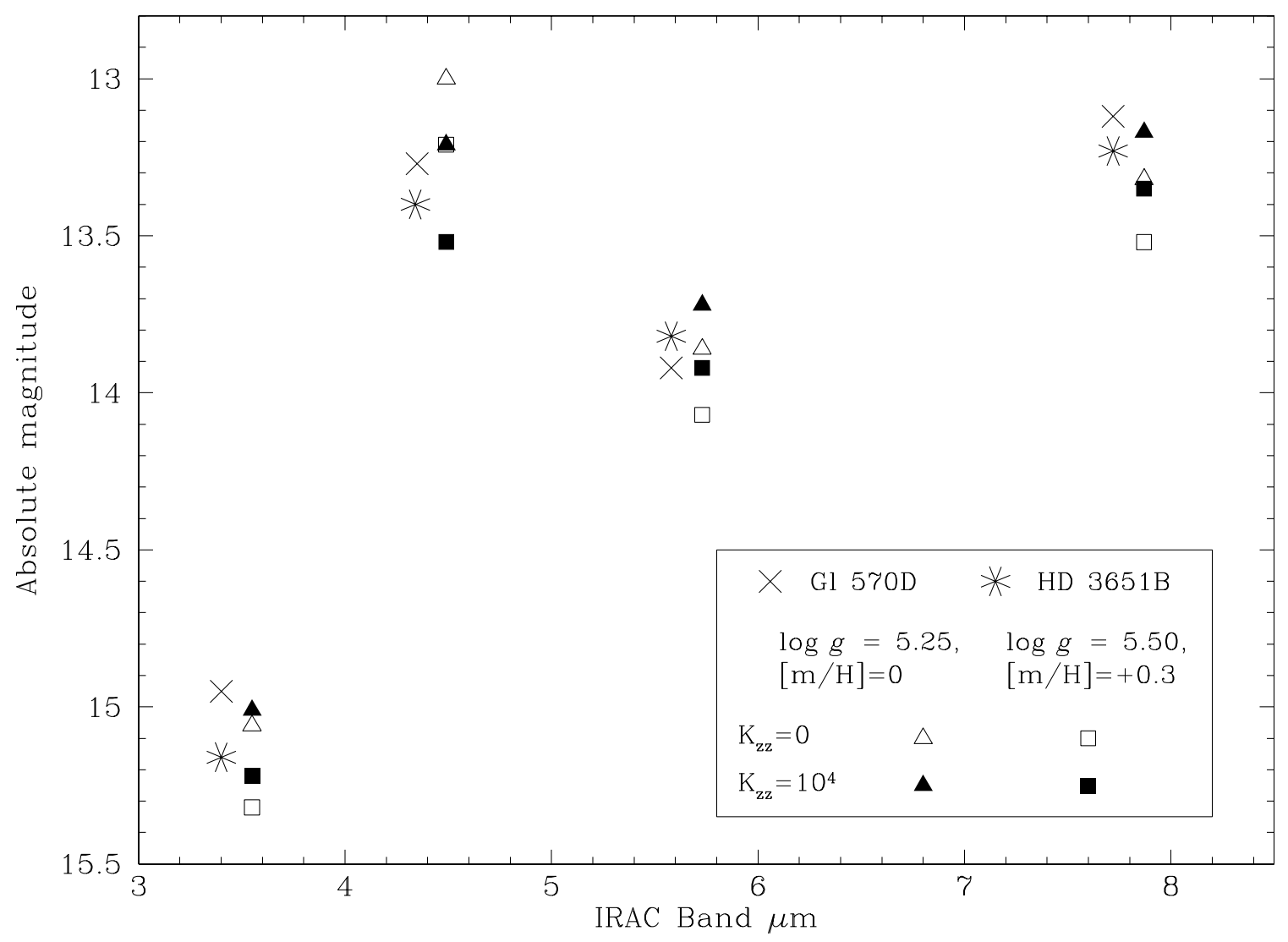

Fig. 8.- Modelled (for $T_{\text {eff }}=825 \mathrm{~K}$ ) and observed absolute IRAC magnitudes, symbols are described in the legend. Observations are taken from Patten et al. (2006) and Luhman et al. (2007); errors in the photometry are $2-5 \%$ for both dwarfs for [3.55] and [4.49], and 7-14\% at [5.73] and [7.87]. Observational datapoints are shifted to shorter wavelengths for clarity. 

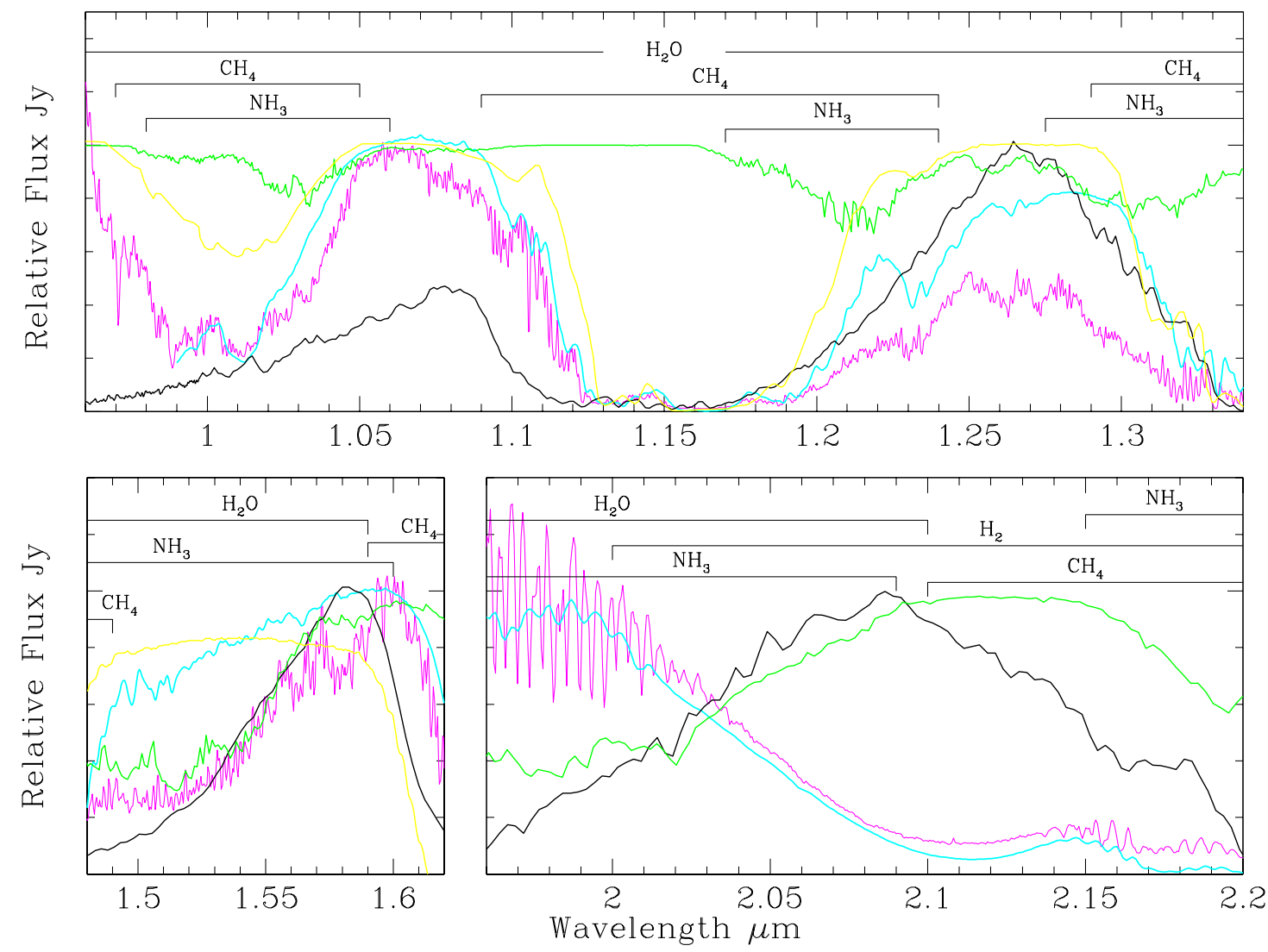

Fig. 9.- Spectra of the T8 dwarf 2MASS0415-09 (black, Knapp et al. 2004), Jupiter (magenta, Rayner, Cushing \& Vacca 2008) and Saturn (cyan, Kim \& Geballe 2005), together with room temperature transmission laboratory data for $\mathrm{CH}_{4}$ (yellow, Cruikshank \& Binder 1968) and $\mathrm{NH}_{3}$ (green, Irwin et al. 1999). 


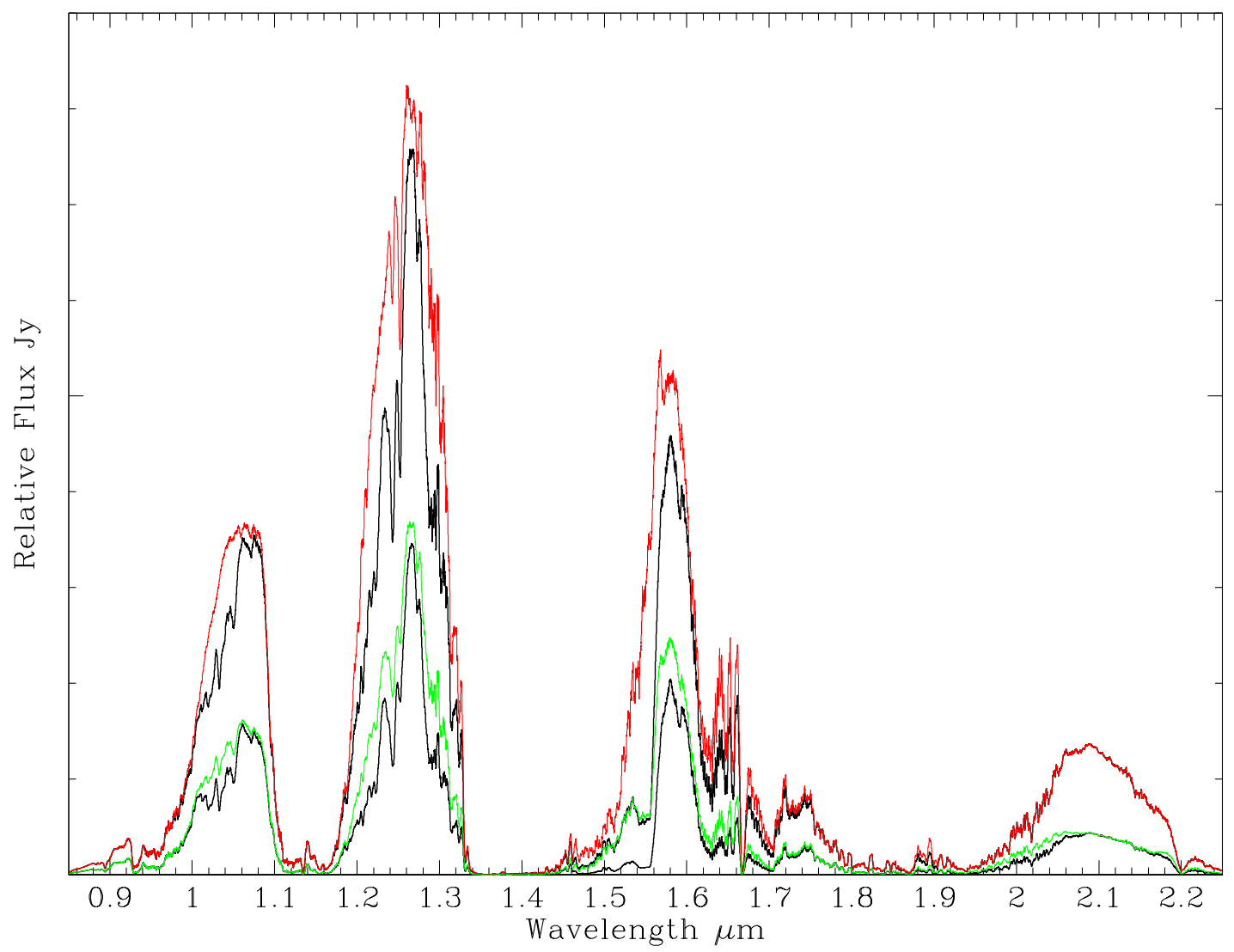

Fig. 10.- Ammonia features in the near-infrared spectra of very cool brown dwarfs. The black curves show cloudless synthetic spectra with $T_{\text {eff }}=700 \mathrm{~K}$ (upper curve) and $600 \mathrm{~K}$ (lower curve), $\log g=5$, and including the $\mathrm{NH}_{3}$ opacity of Irwin et al. (1999) for $\lambda<1.9 \mu \mathrm{m}$ and the $\mathrm{NH}_{3}$ opacity computed from a line list for $\lambda>1.9 \mu \mathrm{m}$. The upper curve (red) shows the $700 \mathrm{~K}$ model computed without the Irwin et al. opacity. A $600 \mathrm{~K}$ model computed with the Irwin et al. opacity but with $\mathrm{NH}_{3}$ depleted by non-equilibrium chemistry (with the eddy diffusion coefficient $K_{z z}=10^{4} \mathrm{~cm}^{2} / \mathrm{s}$ ) is shown by the lower curve (green). All spectra for a given $T_{\text {eff }}$ are computed with the same $(T, P)$ structure. The spectra have been smoothed to a resolving power of $\mathrm{R}=400$. 


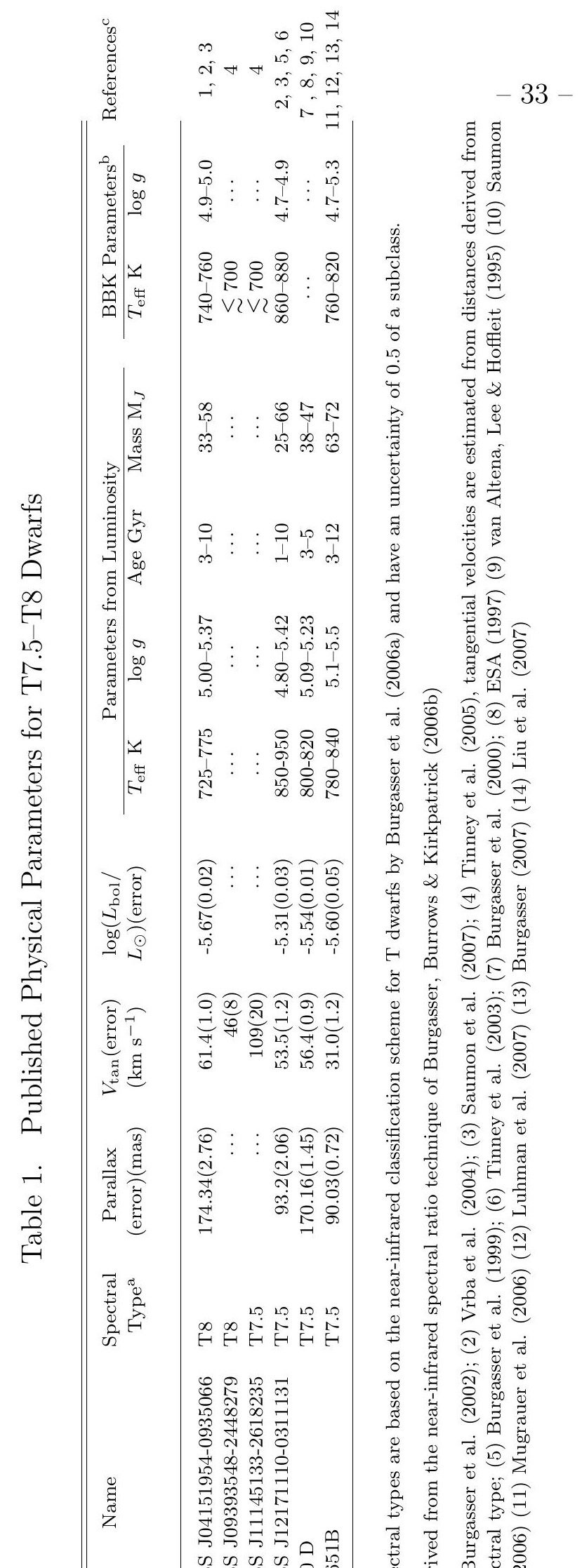


Table 2. Derived Properties of HD 3651B, 2MASS J09393548-2448279 and 2MASS J11145133-2618235

\begin{tabular}{llccccc}
\hline \hline \multicolumn{1}{c}{ Name } & $\begin{array}{c}\text { Spectral } \\
\text { Type }^{\mathrm{a}}\end{array}$ & $\begin{array}{c}\text { Metallicity } \\
{[\mathrm{m} / \mathrm{H}]^{\mathrm{b}}}\end{array}$ & $T_{\text {eff } \mathrm{K}}$ & $\log g^{\mathrm{c}}$ & Age Gyr & ${\text { Mass } \mathrm{M}_{J}{ }^{\mathrm{c}}}$ \\
\hline HD 3651B & $\mathrm{T} 7.5$ & +0.2 & $820-830$ & $5.4-5.5$ & $8-12$ & $60-70$ \\
2MASS J09393548-2448279 & $\mathrm{T} 8$ & -0.3 & $725-775$ & $5.3-5.45$ & $10-12$ & $50-65$ \\
2MASS J11145133-2618235 & $\mathrm{T} 7.5$ & -0.3 & $725-775$ & $5.0-5.3$ & $3-8$ & $30-50$ \\
\hline
\end{tabular}

${ }^{\text {a } U n c e r t a i n t y ~ i n ~ s p e c t r a l ~ t y p e ~ i s ~} 0.5$ of a subclass.

${ }^{\mathrm{b}}$ Uncertainty in metallicity is estimated to be $0.1 \mathrm{dex}$.

${ }^{\mathrm{c}}$ Gravity, age and mass are correlated such that lower gravity implies younger age and lower mass, and higher gravity implies an older and more massive dwarf. 